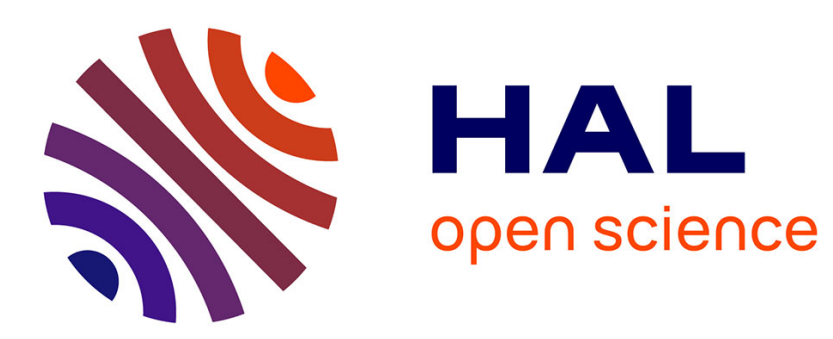

\title{
On the unilateral contact between membranes Part 2: A posteriori analysis and numerical experiments
}

Faker Ben Belgacem, Christine Bernardi, Adel Blouza, Martin Vohralík

\section{To cite this version:}

Faker Ben Belgacem, Christine Bernardi, Adel Blouza, Martin Vohralík. On the unilateral contact between membranes Part 2: A posteriori analysis and numerical experiments. IMA Journal of Numerical Analysis, 2011, 32 (Issue 3), pp.1147-1172. 10.1093/imanum/drr003 . hal-00461144

\section{HAL Id: hal-00461144 \\ https://hal.science/hal-00461144}

Submitted on 4 Mar 2010

HAL is a multi-disciplinary open access archive for the deposit and dissemination of scientific research documents, whether they are published or not. The documents may come from teaching and research institutions in France or abroad, or from public or private research centers.
L'archive ouverte pluridisciplinaire HAL, est destinée au dépôt et à la diffusion de documents scientifiques de niveau recherche, publiés ou non, émanant des établissements d'enseignement et de recherche français ou étrangers, des laboratoires publics ou privés. 


\title{
On the unilateral contact between membranes
}

\author{
Part 2: A posteriori analysis and numerical experiments
}

by Faker Ben Belgacem ${ }^{1}$, Christine Bernardi ${ }^{2}$, Adel Blouza $^{3}$, and Martin Vohralík ${ }^{2}$

\begin{abstract}
The contact between two membranes can be described by a system of variational inequalities, where the unknowns are the displacements of the membranes and the action of a membrane on the other one. A discretization of this system is proposed in Part 1 of this work, where the displacements are approximated by standard finite elements and the action by a local postprocessing which admits an equivalent mixed reformulation. Here, we perform the a posteriori analysis of this discretization and prove optimal error estimates. Next, we present numerical experiments that confirm the efficiency of the error indicators.
\end{abstract}

Résumé: Le contact entre deux membranes peut être décrit par un système d'inéquations variationelles, où les inconnues sont les déplacements des deux membranes et l'action d'une membrane sur l'autre. Une discrétisation de ce système est présentée dans la première partie de ce travail, où les déplacements sont approchés par des éléments finis usuels et l'action par un post-traitement local qui admet une reformulation mixte équivalente. Nous effectuons ici l'analyse a posteriori de cette discrétisation, qui mène à des estimations d'erreur optimales. Puis nous présentons des expériences numériques qui confirment l'efficacité des indicateurs d'erreur.

1 L.M.A.C. (E.A. 2222), Département de Génie Informatique,

Université de Technologie de Compiègne, Centre de Recherches de Royallieu, B.P. 20529, 60205 Compiègne Cedex, France.

e-mail address: faker.ben-belgacem@utc.fr

2 Laboratoire Jacques-Louis Lions, C.N.R.S. \& Université Pierre et Marie Curie,

B.C. 187, 4 place Jussieu, 75252 Paris Cedex 05, France.

e-mail addresses: bernardi@ann.jussieu.fr, vohralik@ann.jussieu.fr

3 Laboratoire de Mathématiques Raphaël Salem (U.M.R. 6085 C.N.R.S.), Université de Rouen, avenue de l'Université, B.P. 12, 76801 Saint-Étienne-du-Rouvray, France.

e-mail address: Adel.Blouza@univ-rouen.fr 



\section{Introduction.}

We are interested in the discretization of the following system, set in a bounded connected open set $\omega$ in $\mathbb{R}^{2}$ with a Lipschitz-continuous boundary:

$$
\begin{cases}-\mu_{1} \Delta u_{1}-\lambda=f_{1} & \text { in } \omega, \\ -\mu_{2} \Delta u_{2}+\lambda=f_{2} & \text { in } \omega, \\ u_{1}-u_{2} \geq 0, \quad \lambda \geq 0, \quad\left(u_{1}-u_{2}\right) \lambda=0 & \text { in } \omega, \\ u_{1}=g & \text { on } \partial \omega \\ u_{2}=0 & \text { on } \partial \omega .\end{cases}
$$

Indeed, such a system is a model for the contact between two membranes and can easily be derived from the fundamental laws of elasticity (more details are given in $[3, \S 2]$ ). In this model, the unknowns are the displacements $u_{1}$ and $u_{2}$ of the two membranes, and the Lagrange multiplier $\lambda$ which represents the action of the second membrane on the first one (equivalently $-\lambda$ represents the action of the first membrane on the second one). The coefficients $\mu_{1}$ and $\mu_{2}$ are positive constants which represent the tensions of the membranes. The data are the external forces $f_{1}$ and $f_{2}$ and also the boundary datum $g$ : Indeed the boundary conditions in system (1.1) mean that the first membrane is fixed on $\partial \omega$ at the height $g$, where $g$ is a nonnegative function, and the second one is fixed at zero.

The analysis of problem (1.1) was first performed in $[3, \S 3]$ in the case $g=0$ of homogeneous boundary conditions (which is simpler but less realistic) and extended to the general case in $[4, \S 2]$. In both situations, two variational problems are considered: A full system satisfied by the three unknowns, made of a variational equality and a variational inequality, and a reduced problem consisting of a variational inequality, where the unknowns are the displacements of the membranes. Relying on these formulations, we have proposed in [4] a discretization made in two steps. In a first step, we introduce a finite element discretization of the reduced problem, prove that the discrete problem is well-posed, and establish optimal a priori estimates under minimal regularity assumptions. The discretization of the full problem relies on the reduced discrete problem but is more complex. It requires the introduction of a dual mesh and can be interpreted as a finite volume scheme. The corresponding discrete problem is well-posed, and optimal a priori error estimates are also derived.

After the pioneering works [13] by Hlaváček, Haslinger, Nečas, and Lovíšek (see Theorem 4.2 in this book) and [1] by Ainsworth, Oden, and Lee, a huge amount of work has been performed on the a posteriori analysis of variational inequalities, see, e.g., [2], [5], [11], [14], [16], [19], [20], and the references therein. It can be noted that, in [11], a mixed problem coupling a variational equality and an inequality is also considered. On the other hand, a way of handling the local nullity of the Laplace operator applied to piecewise affine functions is proposed in [18]; it relies on the introduction of an auxiliary function which can be considered as an interpolate of the gradient of the discrete displacement. By combining these two approaches, we introduce three families of error indicators: The first two ones are linked to the residual of the first two lines of system (1.1), while the third one deals with the equation $\left(u_{1}-u_{2}\right) \lambda=0$ in the third line (indeed, the nonnegativity of the two functions $u_{1}-u_{2}$ and $\lambda$ is preserved by the discrete solution). We thus prove 
optimal a posteriori error estimates. Moreover, since the upper bounds for the indicators are local, we think that they are an efficient tool for mesh adaptivity.

Numerical experiments are performed in two cases: First for a given solution in order to verify the good convergence properties of the discretization, second for unknown solutions when working with simple but illustrative choices of the function $g$.

Acknowledgement: The authors are deeply grateful to Frédéric Hecht for his kind help in using the detailed parts of the code FreeFem++.

An outline of the paper is as follows.

- In Section 2, we recall the variational formulations of system (1.1) and its well-posedness. Next, we describe the discrete problem that is proposed in $[4, \S 3-4]$ and recall a priori error estimates.

- A posteriori error estimates for this discretization are established in Section 3.

- Numerical experiments are presented in Section 4. 


\section{Presentation of the continuous and discrete problems.}

We consider the full scales of Sobolev spaces $H^{s}(\omega)$ and $H^{s}(\partial \omega), s \geq 0$, and, in order to take into account the nonnegativity of the boundary condition $g$, the cones defined by

$$
H_{+}^{s}(\partial \omega)=\left\{k \in H^{s}(\partial \omega) ; k \geq 0 \text { a.e. in } \partial \omega\right\} .
$$

We also need the space $H_{0}^{1}(\omega)$ of functions in $H^{1}(\omega)$ which vanish on $\partial \omega$ and its dual space $H^{-1}(\omega)$. For any function $g$ in $H^{\frac{1}{2}}(\partial \omega)$, we define the space

$$
H_{g}^{1}(\omega)=\left\{v \in H^{1}(\omega) ; v=g \text { on } \partial \omega\right\} .
$$

Next, we introduce the convex subset

$$
\Lambda=\left\{\chi \in L^{2}(\omega) ; \chi \geq 0 \text { a.e. in } \omega\right\} .
$$

So we consider the following variational problem, for any data $\left(f_{1}, f_{2}\right)$ in $H^{-1}(\omega) \times$ $H^{-1}(\omega)$ and $g$ in $H_{+}^{\frac{1}{2}}(\partial \omega)$ :

Find $\left(u_{1}, u_{2}, \lambda\right)$ in $H_{g}^{1}(\omega) \times H_{0}^{1}(\omega) \times \Lambda$ such that

$$
\begin{aligned}
\forall\left(v_{1}, v_{2}\right) \in H_{0}^{1}(\omega) \times H_{0}^{1}(\omega), \quad \sum_{i=1}^{2} \mu_{i} & \int_{\omega}\left(\operatorname{grad} u_{i}\right)(\boldsymbol{x}) \cdot\left(\operatorname{grad} v_{i}\right)(\boldsymbol{x}) \mathrm{d} \boldsymbol{x} \\
& -\int_{\omega} \lambda(\boldsymbol{x})\left(v_{1}-v_{2}\right)(\boldsymbol{x}) \mathrm{d} \boldsymbol{x}=\sum_{i=1}^{2}\left\langle f_{i}, v_{i}\right\rangle,
\end{aligned}
$$

$$
\forall \chi \in \Lambda, \quad \int_{\omega}(\chi-\lambda)(\boldsymbol{x})\left(u_{1}-u_{2}\right)(\boldsymbol{x}) \mathrm{d} \boldsymbol{x} \geq 0,
$$

where $\langle\cdot, \cdot\rangle$ denotes the duality pairing between $H^{-1}(\omega)$ and $H_{0}^{1}(\omega)$. It is readily checked [4, Prop. 1] that problems (1.1) and (2.4) are equivalent. We sum up the main results concerning this problem in the next proposition.

Proposition 2.1. For any data $\left(f_{1}, f_{2}\right)$ in $L^{2}(\omega) \times L^{2}(\omega)$ and $g$ in $H_{+}^{\frac{1}{2}}(\partial \omega)$, problem (2.4) has a unique solution $\left(u_{1}, u_{2}, \lambda\right)$ in $H_{g}^{1}(\omega) \times H_{0}^{1}(\omega) \times \Lambda$. Moreover, this solution satisfies

$$
\left\|u_{1}\right\|_{H^{1}(\omega)}+\left\|u_{2}\right\|_{H^{1}(\omega)}+\|\lambda\|_{L^{2}(\omega)} \leq c\left(\left\|f_{1}\right\|_{L^{2}(\omega)}+\left\|f_{2}\right\|_{L^{2}(\omega)}+\|g\|_{H^{\frac{1}{2}(\partial \omega)}}\right),
$$

and is such that $\left(-\Delta u_{1},-\Delta u_{2}\right)$ belongs to $L^{2}(\omega) \times L^{2}(\omega)$.

As standard for mixed problems, we also introduce the convex set

$$
\mathcal{K}_{g}=\left\{\left(v_{1}, v_{2}\right) \in H_{g}^{1}(\omega) \times H_{0}^{1}(\omega) ; v_{1}-v_{2} \geq 0 \text { a.e. in } \omega\right\},
$$

(since $g$ is nonnegative, this last set is not empty) and consider the reduced problem

Find $\left(u_{1}, u_{2}\right)$ in $\mathcal{K}_{g}$ such that

$$
\begin{array}{r}
\forall\left(v_{1}, v_{2}\right) \in \mathcal{K}_{g}, \quad \sum_{i=1}^{2} \mu_{i} \int_{\omega}\left(\operatorname{grad} u_{i}\right)(\boldsymbol{x}) \cdot\left(\operatorname{grad}\left(v_{i}-u_{i}\right)\right)(\boldsymbol{x}) \mathrm{d} \boldsymbol{x} \\
\geq \sum_{i=1}^{2}\left\langle f_{i}, v_{i}-u_{i}\right\rangle .
\end{array}
$$


Indeed, it can be checked that, for any solution $\left(u_{1}, u_{2}, \lambda\right)$ of problem $(2.4)$, the pair $\left(u_{1}, u_{2}\right)$ is a solution of problem (2.7). So this problem also admits a solution, and its uniqueness is easily derived.

We first describe a discretization of problem (2.7). Assuming that $\omega$ is a polygon, let $\left(\mathcal{T}_{h}\right)_{h}$ be a regular family of triangulations of $\omega$ (by triangles), in the usual sense that:

- For each $h, \bar{\omega}$ is the union of all elements of $\mathcal{T}_{h}$;

- The intersection of two different elements of $\mathcal{T}_{h}$, if not empty, is a vertex or a whole edge of both of them;

- The ratio of the diameter $h_{K}$ of any element $K$ of $\mathcal{T}_{h}$ to the diameter of its inscribed circle is smaller than a constant $\sigma$ independent of $h$.

As usual, $h$ stands for the maximum of the diameters $h_{K}, K \in \mathcal{T}_{h}$. In what follows, $c, c^{\prime}$, ..., stand for generic constants which may vary from line to line but are always independent of $h$.

We use the discrete spaces given as

$$
\mathbb{X}_{h}=\left\{v_{h} \in H^{1}(\omega) ; \forall K \in \mathcal{T}_{h},\left.v_{h}\right|_{K} \in \mathcal{P}_{1}(K)\right\}, \quad \mathbb{X}_{0 h}=\mathbb{X}_{h} \cap H_{0}^{1}(\omega),
$$

where $\mathcal{P}_{1}(K)$ denotes the space of restrictions to $K$ of affine functions.

Next, in order to take into account the nonhomogeneous boundary condition on $u_{1}$, we assume that the datum $g$ belongs to $H^{s+\frac{1}{2}}(\partial \omega)$ for some $s>0$. Thus, we define an approximation $g_{h}$ of $g$ by Lagrange interpolation: The function $g_{h}$ is affine on each edge $e$ of elements of $\mathcal{T}_{h}$ which is contained in $\partial \omega$ and equal to $g$ at each vertex of elements of $\mathcal{T}_{h}$ which belongs to $\partial \omega$. We introduce the affine space

$$
\mathbb{X}_{g h}=\left\{v_{h} \in \mathbb{X}_{h} ; v_{h}=g_{h} \text { on } \partial \omega\right\},
$$

together with the convex set

$$
\mathcal{K}_{g h}=\left\{\left(v_{1 h}, v_{2 h}\right) \in \mathbb{X}_{g h} \times \mathbb{X}_{0 h} ; v_{1 h}-v_{2 h} \geq 0 \text { in } \omega\right\}
$$

The reduced discrete problem is now derived from problem (2.7) by the Galerkin method. It reads:

Find $\left(u_{1 h}, u_{2 h}\right)$ in $\mathcal{K}_{g h}$ such that

$$
\begin{array}{r}
\forall\left(v_{1 h}, v_{2 h}\right) \in \mathcal{K}_{g h}, \quad \sum_{i=1}^{2} \mu_{i} \int_{\omega}\left(\operatorname{grad} u_{i h}\right)(\boldsymbol{x}) \cdot\left(\operatorname{grad}\left(v_{i h}-u_{i h}\right)\right)(\boldsymbol{x}) \mathrm{d} \boldsymbol{x} \\
\geq \sum_{i=1}^{2}\left\langle f_{i}, v_{i h}-u_{i h}\right\rangle .
\end{array}
$$

The next results are proved in [4, Prop. $8 \&$ Thm 9].

Proposition 2.2. For any data $\left(f_{1}, f_{2}\right)$ in $H^{-1}(\omega) \times H^{-1}(\omega)$ and $g$ in $H_{+}^{s+\frac{1}{2}}(\partial \omega), s>0$, problem (2.11) has a unique solution $\left(u_{1 h}, u_{2 h}\right)$ in $\mathcal{K}_{g h}$. If moreover, the domain $\omega$ is convex, the data $\left(f_{1}, f_{2}\right)$ belong to $L^{2}(\omega) \times L^{2}(\omega)$, and the datum $g$ belongs to $H_{+}^{\frac{3}{2}}(\partial \omega)$, the following a priori error estimate holds between the solutions $\left(u_{1}, u_{2}\right)$ of problem $(2.7)$ and $\left(u_{1 h}, u_{2 h}\right)$ of problem $(2.11)$

$$
\left\|u_{1}-u_{1 h}\right\|_{H^{1}(\omega)}+\left\|u_{2}-u_{2 h}\right\|_{H^{1}(\omega)} \leq c h\left(\left\|f_{1}\right\|_{L^{2}(\omega)}+\left\|f_{2}\right\|_{L^{2}(\omega)}+\|g\|_{H^{\frac{3}{2}}(\partial \omega)}\right) .
$$


Let $\mathcal{V}_{h}$ denote the set of vertices of elements of $\mathcal{T}_{h}$ which belong to $\omega$. We thus introduce the Lagrange functions associated with the elements of $\mathcal{V}_{h}$ : For each $\boldsymbol{a}$ in $\mathcal{V}_{h}$, $\varphi_{a}$ belongs to $\mathbb{X}_{0 h}$ and satisfies

$$
\varphi_{\boldsymbol{a}}(\boldsymbol{a})=1 \quad \text { and } \quad \forall \boldsymbol{a}^{\prime} \in \mathcal{V}_{h}, \boldsymbol{a}^{\prime} \neq \boldsymbol{a}, \quad \varphi_{\boldsymbol{a}}\left(\boldsymbol{a}^{\prime}\right)=0 .
$$

We also denote by $\Delta_{\boldsymbol{a}}$ the suport of $\varphi_{\boldsymbol{a}}$ and by $\mathcal{T}_{\boldsymbol{a}}$ the set of elements of $\mathcal{T}_{h}$ that contain $\boldsymbol{a}$.

To describe the full discrete problem, we introduce a new set of nonnegative functions $\chi_{\boldsymbol{a}}, \boldsymbol{a} \in \mathcal{V}_{h}$, as follows: For each $\boldsymbol{a}$ in $\mathcal{V}_{h}$ and with each $K$ in $\mathcal{T}_{\boldsymbol{a}}$, we associate the quadrilateral with vertices $\boldsymbol{a}$, the midpoints of the two edges of $K$ that contain $\boldsymbol{a}$, and the barycentre of $K$ and we denote by $D_{\boldsymbol{a}}$ the union of these quadrilaterals when $K$ runs through $\mathcal{T}_{\boldsymbol{a}}$ (see [4, Fig. 1] for more details); each function $\chi_{\boldsymbol{a}}$ is then taken equal to the characteristic function of $D_{a}$.

Let $\mathcal{D}_{h}$ be the set of the $D_{\boldsymbol{a}}, \boldsymbol{a} \in \mathcal{V}_{h}$, and $\mathbb{Y}_{h}$ be the space spanned by the $\chi_{\boldsymbol{a}}$. Thus, it is readily checked that the set

$$
\Lambda_{h}=\left\{\mu_{h}=\sum_{\boldsymbol{a} \in \mathcal{V}_{h}} \mu_{\boldsymbol{a}} \chi_{\boldsymbol{a}} ; \mu_{\boldsymbol{a}} \geq 0\right\}
$$

is a convex cone contained in $\Lambda$. We also introduce a duality pairing between $\mathbb{Y}_{h}$ and $\mathbb{X}_{h}$ by

$$
\begin{aligned}
\forall \mu_{h}=\sum_{\boldsymbol{a} \in \mathcal{V}_{h}} \mu_{\boldsymbol{a}} \chi_{\boldsymbol{a}} \in \mathbb{Y}_{h}, \forall v_{h} \in \mathbb{X}_{0 h}, & \\
\left\langle\mu_{h}, v_{h}\right\rangle_{h} & =\sum_{\boldsymbol{a} \in \mathcal{V}_{h}} \mu_{\boldsymbol{a}} v_{h}(\boldsymbol{a}) \sum_{K \in \mathcal{T}_{\boldsymbol{a}}} \int_{K} \varphi_{\boldsymbol{a}}(\boldsymbol{x}) \mathrm{d} \boldsymbol{x} .
\end{aligned}
$$

We can now define functions $\lambda_{1 h}$ and $\lambda_{2 h}$ in $\mathbb{Y}_{h}$ by the equations, where $v_{1 h}$ and $v_{2 h}$ run through $\mathbb{X}_{0 h}$,

$$
\begin{aligned}
& \left\langle\lambda_{1 h}, v_{1 h}\right\rangle_{h}=\mu_{1} \int_{\omega}\left(\operatorname{grad} u_{1 h}\right)(\boldsymbol{x}) \cdot\left(\operatorname{grad} v_{1 h}\right)(\boldsymbol{x}) \mathrm{d} \boldsymbol{x}-\int_{\omega} f_{1}(\boldsymbol{x}) v_{1 h}(\boldsymbol{x}) \mathrm{d} \boldsymbol{x}, \\
& \left\langle\lambda_{2 h}, v_{2 h}\right\rangle_{h}=-\mu_{2} \int_{\omega}\left(\operatorname{grad} u_{2 h}\right)(\boldsymbol{x}) \cdot\left(\operatorname{grad} v_{2 h}\right)(\boldsymbol{x}) \mathrm{d} \boldsymbol{x}+\int_{\omega} f_{2}(\boldsymbol{x}) v_{2 h}(\boldsymbol{x}) \mathrm{d} \boldsymbol{x} .
\end{aligned}
$$

It can be checked [4, Prop. 12] that the functions $\lambda_{1 h}$ and $\lambda_{2 h}$ defined in (2.16) coincide.

The full discrete problem reads

Find $\left(u_{1 h}, u_{2 h}, \lambda_{h}\right)$ in $\mathbb{X}_{g h} \times \mathbb{X}_{0 h} \times \Lambda_{h}$ such that

$$
\begin{array}{r}
\forall\left(v_{1 h}, v_{2 h}\right) \in \mathbb{X}_{0 h} \times \mathbb{X}_{0 h}, \quad \sum_{i=1}^{2} \mu_{i} \int_{\omega}\left(\operatorname{grad} u_{i h}\right)(\boldsymbol{x}) \cdot\left(\operatorname{grad} v_{i h}\right)(\boldsymbol{x}) \mathrm{d} \boldsymbol{x} \\
-\left\langle\lambda_{h}, v_{1 h}-v_{2 h}\right\rangle_{h}=\sum_{i=1}^{2}\left\langle f_{i}, v_{i h}\right\rangle,
\end{array}
$$

$\forall \chi_{h} \in \Lambda_{h}, \quad\left\langle\chi_{h}-\lambda_{h}, u_{1 h}-u_{2 h}\right\rangle_{h} \geq 0$.

The next results are proved in $[4, \S 4]$. 
Proposition 2.3. For any data $\left(f_{1}, f_{2}\right)$ in $L^{2}(\omega) \times L^{2}(\omega)$ and $g$ in $H_{+}^{s+\frac{1}{2}}(\partial \omega), s>0$, problem (2.17) has a unique solution $\left(u_{1 h}, u_{2 h}, \lambda_{h}\right)$ in $\mathbb{X}_{g h} \times \mathbb{X}_{0 h} \times \Lambda_{h}$. Moreover,

(i) For any solution $\left(u_{1 h}, u_{2 h}, \lambda_{h}\right)$ of problem (2.17), the pair $\left(u_{1 h}, u_{2 h}\right)$ is a solution of problem (2.11);

(ii) For any solution $\left(u_{1 h}, u_{2 h}\right)$ of problem $(2.11)$, the function $\lambda_{h}=\lambda_{i h}, i=1,2$, defined in (2.16) gives rise to a solution $\left(u_{1 h}, u_{2 h}, \lambda_{h}\right)$ of problem $(2.17)$.

If the assumptions of Proposition 2.2 are satisfied and if there exists a constant $\sigma$ independent of $h$ such that

$$
\forall K \in \mathcal{T}_{h}, \quad h_{K} \geq \sigma h,
$$

the following a priori error estimate holds between the solutions $\left(u_{1}, u_{2}, \lambda\right)$ of problem $(2.4)$ and $\left(u_{1 h}, u_{2 h}, \lambda_{h}\right)$ of problem $(2.17)$

$$
\left\|\lambda-\lambda_{h}\right\|_{H^{-1}(\omega)} \leq c h\left(\left\|f_{1}\right\|_{L^{2}(\omega)}+\left\|f_{2}\right\|_{L^{2}(\omega)}+\|g\|_{H^{\frac{3}{2}(\partial \omega)}}\right) .
$$

Estimates (2.12) and (2.19) are fully optimal. However, assumption (2.18) is very restrictive in the context of mesh adaptivity. Fortunately, if it is replaced by the more realistic one

$$
\forall K \in \mathcal{T}_{h}, \quad h_{K} \geq \sigma h^{1+\alpha},
$$

for a real number $\alpha, 0<\alpha<1$, the same arguments as previously yield a convergence of $\left\|\lambda-\lambda_{h}\right\|_{H^{-1}(\omega)}$ of order $h^{1-\alpha}$.

It can also be noted that the reduced problem (2.11) provides a natural algorithm for uncoupling the unknowns and, once its solution $\left(u_{1 h}, u_{2 h}\right)$ is known, computing $\lambda_{h}$ consists in solving a linear system with diagonal matrix, see (2.16). 


\section{A posteriori analysis.}

We first introduce some further notation and define the three types of error indicators which are needed for our analysis. Next, we prove successively upper bounds of the error as a function of the indicators and upper bounds of the indicators as a function of the error. We conclude with a remark on the optimality of these results.

\subsection{Some notation and the error indicators.}

In order to describe the error indicators, we need a further notation. We first introduce the set $\overline{\mathcal{V}}_{h}$ of all vertices of elements of $\mathcal{T}_{h}$. With each vertex $\boldsymbol{a}$ in $\overline{\mathcal{V}}_{h}$ which does not belong to $\mathcal{V}_{h}$, we associate a polygon $D_{\boldsymbol{a}}$ defined exactly as in Section 2 . The set of all $D_{\boldsymbol{a}}, \boldsymbol{a} \in \overline{\mathcal{V}}_{h}$, is denoted by $\overline{\mathcal{D}}_{h}$. With each triangulation $\mathcal{T}_{h}$, we also associate the triangulation $\mathcal{S}_{h}$ built in the following way: Any triangle $K$ in $\mathcal{T}_{h}$ is divided into six "small" triangles $\kappa$ by joining the barycentre of $K$ to its vertices and the middle of its edges, and these $\kappa$ form the new triangulation $\mathcal{S}_{h}$.

Let $H(\operatorname{div}, \omega)$ denote the domain of the divergence operator, namely the space of functins $s$ in $L^{2}(\omega)^{2}$ such that div $s$ belongs to $L^{2}(\omega)$. On this triangulation $\mathcal{S}_{h}$, we define the space associated with Raviart-Thomas finite elements [15]

$$
\mathbb{Z}_{h}=\left\{s_{h} \in H(\operatorname{div}, \omega) ; \forall \kappa \in \mathcal{S}_{h},\left.s_{h}\right|_{\kappa} \in \mathcal{R} \mathcal{T}(\kappa)\right\}
$$

where $\mathcal{R} \mathcal{T}(\kappa)$ stands for the space of restrictions to $\kappa$ of polynomials of the form $\boldsymbol{c}+d \boldsymbol{x}$, $\boldsymbol{c} \in \mathbb{R}^{2}, d \in \mathbb{R}$. We recall from [15] that the linear forms: $\boldsymbol{s} \mapsto \int_{e}(\boldsymbol{s} \cdot \boldsymbol{n})(\tau) \mathrm{d} \tau$ where $\boldsymbol{n}$ is a unit normal vector to $e$ and $e$ runs through the edges of $\kappa$, are $\mathcal{R} \mathcal{T}(\kappa)$-unisolvent and that the functions of $\mathbb{Z}_{h}$ have a constant normal trace on each edge of elements of $\mathcal{S}_{h}$. We denote by $\mathcal{E}_{h}$ the set of all edges of the $\kappa$ in $\mathcal{S}_{h}$, and by $\mathcal{E}_{h}^{*}$ the set of edges of the $\kappa$ which are inside an element $K$ of $\mathcal{T}_{h}$.

Following the approach in [18], we introduce two auxiliary unknowns $\boldsymbol{t}_{i h}$ in $\mathbb{Z}_{h}$ satisfying on each edge $e$ of $\mathcal{E}_{h}^{*}$ which is inside an element $K$ of $\mathcal{T}_{h}$

$$
\left.\left(\boldsymbol{t}_{i h} \cdot \boldsymbol{n}\right)\right|_{e}=-\mu_{i} \partial_{n}\left(\left.u_{i h}\right|_{K}\right)
$$

where $\boldsymbol{n}$ denotes one of the unit normal vectors to $e$. Of course, these conditions are not sufficient to define the $\boldsymbol{t}_{i h}$ in a unique way. However, we prefer to give the complete definition later on.

Denoting by $h_{D}$ the diameter of each $D$ in $\overline{\mathcal{D}}_{h}$, we introduce the following constants: (i) For each $D$ in $\overline{\mathcal{D}}_{h}$, the Poincaré-Wirtinger constant $c_{D}^{(P W)}$ is the smallest constant such that

$$
\forall \varphi \in H^{1}(D), \quad\left\|\varphi-\bar{\varphi}^{D}\right\|_{L^{2}(D)} \leq c_{D}^{(P W)} h_{D}\|\operatorname{grad} \varphi\|_{L^{2}(D)^{2}},
$$

where $\bar{\varphi}^{D}$ stands for the mean value of $\varphi$ on $D$;

(ii) For each $D$ in $\overline{\mathcal{D}}_{h} \backslash \mathcal{D}_{h}$, the Poincaré-Friedrichs constant $c_{D}^{(P F)}$ is the smallest constant such that

$$
\forall \varphi \in H_{*}^{1}(D), \quad\|\varphi\|_{L^{2}(D)} \leq c_{D}^{(P F)} h_{D}\|\operatorname{grad} \varphi\|_{L^{2}(D)^{2}},
$$

where $H_{*}^{1}(D)$ denotes the space of functions in $H^{1}(D)$ which vanish on $\partial D \cap \partial \omega$.

We denote by $c_{D}$ the constant $c_{D}^{(P F)}$ if $D$ belongs to $\overline{\mathcal{D}}_{h} \backslash \mathcal{D}_{h}$ and the constant $c_{D}^{(P W)}$ if $D$ belongs to $\mathcal{D}_{h}$. 
We also consider an approximation $f_{i h}$ of each function $f_{i}$ in the space

$$
\mathbb{F}_{h}=\left\{f_{h} \in L^{2}(\omega) ; \forall K \in \mathcal{T}_{h},\left.f_{h}\right|_{K} \in \mathcal{P}_{0}(K)\right\},
$$

where $\mathcal{P}_{0}(K)$ is the space of restrictions to $K$ of constant functions. More precisely, for each triangle $K$ in $\mathcal{T}_{h},\left.f_{i h}\right|_{K}$ is equal to the mean value of $f_{i}$ on $K$. Next, we define three types of error indicators, for each $D$ in $\overline{\mathcal{D}}_{h}$ and for $i=1,2$ :

(i) The diffusive flux estimators $\eta_{i D}^{(\mathrm{df})}$

$$
\eta_{i D}^{(\mathrm{df})}=\left\|\mu_{i}^{\frac{1}{2}} \operatorname{grad} u_{i h}+\mu_{i}^{-\frac{1}{2}} \boldsymbol{t}_{i h}\right\|_{L^{2}(D)^{2}}
$$

(ii) The residual error estimators $\eta_{i D}^{(\mathrm{r})}$

$$
\eta_{i D}^{(\mathrm{r})}=c_{D} h_{D} \mu_{i}^{-\frac{1}{2}}\left\|f_{i h}-\operatorname{div} \boldsymbol{t}_{i h}-(-1)^{i} \lambda_{h}\right\|_{L^{2}(D)} .
$$

(iii) The indicators $\eta_{D}^{(\mathrm{c})}$ related to the contact equation

$$
\eta_{D}^{(\mathrm{c})}=2 \int_{D}\left(u_{1 h}-u_{2 h}\right)(\boldsymbol{x}) \lambda_{h}(\boldsymbol{x}) d \boldsymbol{x}
$$

It can be noted that, despite the introduction of auxiliary unknowns, all these indicators are easy to compute since they only involve constant or affine functions.

Remark 3.1. Let $\omega^{c}$ denote the contact zone, i.e., the set of points in $\bar{\omega}$ where $u_{1}-u_{2}$ vanishes. We also define the discrete contact zone $\omega_{h}^{c}$ as the union of the elements $K$ in $\mathcal{T}_{h}$ such that $u_{1 h}-u_{2 h}$ vanishes at the three vertices of $K$. On the other hand, we recall from $\left[4\right.$, Form. (4.8)] that, with the notation $\lambda_{h}=\sum_{\boldsymbol{a} \in \mathcal{V}_{h}} \lambda_{\boldsymbol{a}} \chi_{\boldsymbol{a}}$,

$$
\forall \boldsymbol{a} \in \mathcal{V}_{h}, \quad \lambda_{\boldsymbol{a}}\left(u_{1 h}-u_{2 h}\right)(\boldsymbol{a})=0
$$

So, the $D_{\boldsymbol{a}}$ such that $\eta_{D_{\boldsymbol{a}}}^{(\mathrm{c})}$ is not zero are such that $u_{1 h}-u_{2 h}$ vanishes at $\boldsymbol{a}$ but not at all vertices of $\Delta_{a}$ (this corresponds to the standard choice of the quadrature formula). Equivalently, such $D_{a}$ are contained in a small neighbourhood of the boundary of $\omega_{h}^{c}$, hence are not so many. A family of indicators which are similar to the $\eta_{D}^{(\mathrm{c})}$ is introduced in $[19, \S 4]$ and considered as negligible.

In order to prove the first a posteriori error estimate, we introduce the energy seminorm, for any pair $\boldsymbol{v}=\left(v_{1}, v_{2}\right)$ in $H^{1}(\omega)^{2}$,

$$
\|\boldsymbol{v}\|=\left(\mu_{1}\left|v_{1}\right|_{H^{1}(\omega)}^{2}+\mu_{2}\left|v_{2}\right|_{H^{1}(\omega)}^{2}\right)^{\frac{1}{2}} .
$$

To simplify the next proofs, setting $\boldsymbol{u}=\left(u_{1}, u_{2}\right)$ and $\boldsymbol{v}=\left(v_{1}, v_{2}\right)$, we also consider the bilinear forms

$$
a(\boldsymbol{u}, \boldsymbol{v})=\sum_{i=1}^{2} \mu_{i} \int_{\omega}\left(\operatorname{grad} u_{i}\right)(\boldsymbol{x}) \cdot\left(\operatorname{grad} v_{i}\right)(\boldsymbol{x}) \mathrm{d} \boldsymbol{x}
$$

and

$$
b(\boldsymbol{v}, \chi)=-\int_{\omega} \chi(\boldsymbol{x})\left(v_{1}-v_{2}\right)(\boldsymbol{x}) \mathrm{d} \boldsymbol{x} .
$$




\subsection{Upper bounds for the error.}

The idea to handle the nonhomogeneous boundary condition consists in introducing the solution $\left(\tilde{u}_{1}, \tilde{u}_{2}, \tilde{\lambda}\right)$ of problem $(2.4)$ with $g$ replaced by $g_{h}$. Indeed, the main error estimate relies on the triangle inequality

$$
\left\|\boldsymbol{u}-\boldsymbol{u}_{h}\right\| \leq\|\boldsymbol{u}-\tilde{\boldsymbol{u}}\|+\left\|\tilde{\boldsymbol{u}}-\boldsymbol{u}_{h}\right\|
$$

We also denote by $H_{*}^{\frac{1}{2}}(\partial \omega)$ the set of functions in $L^{2}(\partial \omega)$ such that their restrictions to each edge $\gamma$ of $\partial \omega$ belong to $H^{\frac{1}{2}}(\gamma)$ and by $H_{*}^{-\frac{1}{2}}(\partial \omega)$ its dual space.

Lemma 3.2. For any data $\left(f_{1}, f_{2}\right)$ in $L^{2}(\omega) \times L^{2}(\omega)$, if the domain $\omega$ is convex, the following estimate holds

$$
\|\boldsymbol{u}-\tilde{\boldsymbol{u}}\| \leq c\left(\left\|g-g_{h}\right\|_{H^{\frac{1}{2}}(\partial \omega)}+\sqrt{\rho\left(f_{1}, f_{2}\right)}\left\|g-g_{h}\right\|_{H_{*}^{-\frac{1}{2}}(\partial \omega)}^{\frac{1}{2}}\right),
$$

where the constant $\rho\left(f_{1}, f_{2}\right)$ is given by

$$
\rho\left(f_{1}, f_{2}\right)=\left\|f_{1}\right\|_{L^{2}(\omega)}+\left\|f_{2}\right\|_{L^{2}(\omega)} .
$$

Proof: We proceed in two steps.

1) Let $w_{1}$ be the harmonic lifting of the function $g-g_{h}$; it satisfies

$$
\left|w_{1}\right|_{H^{1}(\omega)} \leq c\left\|g-g_{h}\right\|_{H^{\frac{1}{2}}(\partial \omega)} .
$$

Moreover, we have

$$
\left\|w_{1}\right\|_{L^{2}(\omega)}=\sup _{\varphi \in L^{2}(\omega)} \frac{\int_{\omega} w_{1}(\boldsymbol{x}) \varphi(\boldsymbol{x}) \mathrm{d} \boldsymbol{x}}{\|\varphi\|_{L^{2}(\omega)}} .
$$

Since $\omega$ is a convex polygon, for any $\varphi$ in $L^{2}(\omega)$, the solution $\psi$ of the equation

$$
-\Delta \psi=\varphi \quad \text { in } \omega, \quad \psi=0 \quad \text { on } \partial \omega
$$

belongs to $H^{2}(\omega)$ and satisfies

$$
\left\|\partial_{n} \psi\right\|_{H_{*}^{\frac{1}{2}}(\partial \omega)} \leq c\|\varphi\|_{L^{2}(\omega)} .
$$

Moreover, by integrating twice by parts, we see that

$$
\int_{\omega} w_{1}(\boldsymbol{x}) \varphi(\boldsymbol{x}) \mathrm{d} \boldsymbol{x}=-\int_{\partial \omega}\left(g-g_{h}\right)(\tau)\left(\partial_{n} \psi\right)(\tau) \mathrm{d} \tau .
$$

Combining this line with (3.17) and (3.18), we derive

$$
\left\|w_{1}\right\|_{L^{2}(\omega)} \leq c\left\|g-g_{h}\right\|_{H_{*}^{-\frac{1}{2}}(\partial \omega)} .
$$

2 ) We take $\boldsymbol{w}$ equal to $\left(w_{1}, 0\right)$. Then $\boldsymbol{u}-\tilde{\boldsymbol{u}}-\boldsymbol{w}$ belongs to $H_{0}^{1}(\omega)^{2}$. Applying twice problem (2.4) with $\boldsymbol{v}$ equal to $\boldsymbol{u}-\tilde{\boldsymbol{u}}-\boldsymbol{w}$ yields

$$
a(\boldsymbol{u}-\tilde{\boldsymbol{u}}, \boldsymbol{u}-\tilde{\boldsymbol{u}}) \leq a(\boldsymbol{u}-\tilde{\boldsymbol{u}}, \boldsymbol{w})-b(\boldsymbol{u}-\tilde{\boldsymbol{u}}-\boldsymbol{w}, \lambda-\tilde{\lambda}) .
$$


We also have

$$
b(\boldsymbol{u}, \lambda)=b(\tilde{\boldsymbol{u}}, \tilde{\lambda})=0, \quad b(\boldsymbol{u}, \tilde{\lambda}) \leq 0, \quad b(\tilde{\boldsymbol{u}}, \lambda) \leq 0,
$$

whence

$$
a(\boldsymbol{u}-\tilde{\boldsymbol{u}}, \boldsymbol{u}-\tilde{\boldsymbol{u}}) \leq a(\boldsymbol{u}-\tilde{\boldsymbol{u}}, \boldsymbol{w})+b(\boldsymbol{w}, \lambda-\tilde{\lambda}) .
$$

By two Cauchy-Schwarz inequalities, this gives

$$
\|\boldsymbol{u}-\tilde{\boldsymbol{u}}\|^{2} \leq\|\boldsymbol{u}-\tilde{\boldsymbol{u}}\| \mu_{1}^{\frac{1}{2}}\left|w_{1}\right|_{H^{1}(\Omega)}+\|\lambda-\tilde{\lambda}\|_{L^{2}(\omega)}\left\|w_{1}\right\|_{L^{2}(\omega)} .
$$

We recall from [4, Prop. 4] that

$$
\|\lambda\|_{L^{2}(\omega)}+\|\tilde{\lambda}\|_{L^{2}(\omega)} \leq c \rho\left(f_{1}, f_{2}\right) .
$$

So, the desired estimate is now a direct consequence of the previous two inequalities, combined with (3.16) and (3.19).

When $\omega$ is not convex, estimate (3.14) still holds, but with $\left\|g-g_{h}\right\|_{H_{*}^{-\frac{1}{2}}(\partial \omega)}$ replaced by $\left\|g-g_{h}\right\|_{H^{-\alpha}(\partial \omega)}$ for some $\alpha, 0<\alpha<\frac{1}{2}$. In any case this estimate is optimal when the datum $g$ is smooth. A different way for handling this nonhomogeneous boundary condition is proposed in $[6, \S 2]$ but requires the further assumption $g \leq g_{h}$ on $\partial \omega$ that we prefer to avoid here.

We now prove the bound for the second term in (3.13).

Lemma 3.3. Assume that the data $\left(f_{1}, f_{2}\right)$ belong to $L^{2}(\omega) \times L^{2}(\omega)$. Then, the following estimate holds

$$
\left\|\tilde{\boldsymbol{u}}-\boldsymbol{u}_{h}\right\| \leq\left(\sum_{D \in \overline{\mathcal{D}}_{h}}\left(\sum_{i=1}^{2}\left(\eta_{i D}^{(\mathrm{df})}+\eta_{i D}^{(\mathrm{r})}+c \mu_{i}^{-\frac{1}{2}} h_{D}\left\|f_{i}-f_{i h}\right\|_{L^{2}(D)}\right)^{2}+\eta_{D}^{(\mathrm{c})}\right)\right)^{\frac{1}{2}} .
$$

Proof: Setting $\boldsymbol{\zeta}=\tilde{\boldsymbol{u}}-\boldsymbol{u}_{h}$ and using notation (3.10), we have

$$
\left\|\tilde{\boldsymbol{u}}-\boldsymbol{u}_{h}\right\|^{2}=a\left(\tilde{\boldsymbol{u}}-\boldsymbol{u}_{h}, \boldsymbol{\zeta}\right)
$$

Since $\boldsymbol{\zeta}$ vanishes on $\partial \omega$, we thus derive from the analogue of problem (2.7) that

$$
a\left(\tilde{\boldsymbol{u}}-\boldsymbol{u}_{h}, \boldsymbol{\zeta}\right) \leq \sum_{i=1}^{2} \int_{\omega} f_{i}(\boldsymbol{x}) \zeta_{i}(\boldsymbol{x}) \mathrm{d} \boldsymbol{x}-a\left(\boldsymbol{u}_{h}, \boldsymbol{\zeta}\right)
$$

whence

$$
a\left(\tilde{\boldsymbol{u}}-\boldsymbol{u}_{h}, \boldsymbol{\zeta}\right) \leq \sum_{i=1}^{2} \int_{\omega}\left(f_{i}-(-1)^{i} \lambda_{h}\right)(\boldsymbol{x}) \zeta_{i}(\boldsymbol{x}) \mathrm{d} \boldsymbol{x}-a\left(\boldsymbol{u}_{h}, \boldsymbol{\zeta}\right)+b\left(\boldsymbol{\zeta}, \lambda_{h}\right) .
$$

Noting that $\boldsymbol{\zeta}$ vanishes on $\partial \omega$, inserting the equation

$$
\int_{\omega}\left(\operatorname{div} \boldsymbol{t}_{i h}\right)(\boldsymbol{x}) \zeta_{i}(\boldsymbol{x}) \mathrm{d} \boldsymbol{x}=-\int_{\omega} \boldsymbol{t}_{i h}(\boldsymbol{x}) \cdot\left(\operatorname{grad} \zeta_{i}\right)(\boldsymbol{x}) \mathrm{d} \boldsymbol{x}
$$


and using the nonpositivity of $b\left(\tilde{\boldsymbol{u}}, \lambda_{h}\right)$ ( since $\lambda_{h}$ is nonnegative), we obtain

$$
\begin{aligned}
a\left(\tilde{\boldsymbol{u}}-\boldsymbol{u}_{h}, \boldsymbol{\zeta}\right) \leq & \sum_{i=1}^{2}\left(\int_{\omega}\left(f_{i h}-\operatorname{div} \boldsymbol{t}_{i h}-(-1)^{i} \lambda_{h}\right)(\boldsymbol{x}) \zeta_{i}(\boldsymbol{x}) \mathrm{d} \boldsymbol{x}+\int_{\omega}\left(f_{i}-f_{i h}\right)(\boldsymbol{x}) \zeta_{i}(\boldsymbol{x}) \mathrm{d} \boldsymbol{x}\right. \\
& \left.-\int_{\omega}\left(\mu_{i}^{\frac{1}{2}} \operatorname{grad} \boldsymbol{u}_{i h}+\mu_{i}^{-\frac{1}{2}} \boldsymbol{t}_{i h}\right)(\boldsymbol{x}) \cdot \mu_{i}^{\frac{1}{2}}\left(\operatorname{grad} \zeta_{i}\right)(\boldsymbol{x}) \mathrm{d} \boldsymbol{x}\right)-b\left(\boldsymbol{u}_{h}, \lambda_{h}\right) .
\end{aligned}
$$

We now write the first and third integrals in this last inequality as a sum of integrals on the $D$ in $\overline{\mathcal{D}}_{h}$. We evaluate successively these integrals.

1) Using the orthogonality of $f_{i}-f_{i h}$ to the constants on all $K$ in $\mathcal{T}_{h}$ yields

$$
\int_{\omega}\left(f_{i}-f_{i h}\right)(\boldsymbol{x}) \zeta_{i}(\boldsymbol{x}) \mathrm{d} \boldsymbol{x}=\int_{\omega}\left(f_{i}-f_{i h}\right)(\boldsymbol{x})\left(\zeta_{i}-\zeta_{i h}\right)(\boldsymbol{x}) \mathrm{d} \boldsymbol{x}
$$

where $\zeta_{i h}$ stands for the approximation of $\zeta_{i}$ which is constant on each $K$ in $\mathcal{T}_{h}$ equal to the mean value of $\zeta_{i}$ on $K$. Thus, standard approximation properties [7, Thm 3.1.6] lead to

$$
\int_{\omega}\left(f_{i}-f_{i h}\right)(\boldsymbol{x}) \zeta_{i}(\boldsymbol{x}) \mathrm{d} \boldsymbol{x} \leq c \mu_{i}^{-\frac{1}{2}} \sum_{K \in \mathcal{T}_{h}} h_{K}\left\|f_{i}-f_{i h}\right\|_{L^{2}(K)}\left\|\mu_{i}^{\frac{1}{2}} \operatorname{grad} \zeta_{i}\right\|_{L^{2}(K)^{2}}
$$

whence

$$
\int_{\omega}\left(f_{i}-f_{i h}\right)(\boldsymbol{x}) \zeta_{i}(\boldsymbol{x}) \mathrm{d} \boldsymbol{x} \leq c \mu_{i}^{-\frac{1}{2}} \sum_{D \in \overline{\mathcal{D}}_{h}} h_{D}\left\|f_{i}-f_{i h}\right\|_{L^{2}(D)}\left\|\mu_{i}^{\frac{1}{2}} \operatorname{grad} \zeta_{i}\right\|_{L^{2}(D)^{2}}
$$

2) For all $D$ in $\overline{\mathcal{D}}_{h}$, we derive from the Cauchy-Schwarz inequality that

$$
\begin{aligned}
\int_{D}\left(\mu_{i}^{\frac{1}{2}} \operatorname{grad} \boldsymbol{u}_{i h}\right. & \left.+\mu_{i}^{-\frac{1}{2}} \boldsymbol{t}_{i h}\right)(\boldsymbol{x}) \cdot \mu_{i}^{\frac{1}{2}}\left(\operatorname{grad} \zeta_{i}\right)(\boldsymbol{x}) \mathrm{d} \boldsymbol{x} \\
& \leq\left\|\mu_{i}^{\frac{1}{2}} \operatorname{grad} \boldsymbol{u}_{i h}+\mu_{i}^{-\frac{1}{2}} \boldsymbol{t}_{i h}\right\|_{L^{2}(D)^{2}}\left\|\mu_{i}^{\frac{1}{2}} \operatorname{grad} \zeta_{i}\right\|_{L^{2}(D)^{2}} .
\end{aligned}
$$

3) For all $D$ in $\overline{\mathcal{D}}_{h} \backslash \mathcal{D}_{h}$, by combining the Poincaré-Friedrichs inequality (3.4) with the Cauchy-Schwarz inequality, we obtain

$$
\begin{aligned}
& \int_{D}\left(f_{i h}-\operatorname{div} \boldsymbol{t}_{i h}-(-1)^{i} \lambda_{h}\right)(\boldsymbol{x}) \zeta_{i}(\boldsymbol{x}) \mathrm{d} \boldsymbol{x} \\
& \leq c_{D}^{(P F)} h_{D} \mu_{i}^{-\frac{1}{2}}\left\|f_{i h}-\operatorname{div} \boldsymbol{t}_{i h}-(-1)^{i} \lambda_{h}\right\|_{L^{2}(D)}\left\|\mu_{i}^{\frac{1}{2}} \operatorname{grad} \zeta_{i}\right\|_{L^{2}(D)^{2}} .
\end{aligned}
$$

4) A further argument is needed to handle this same integral on the elements $D$ in $\mathcal{D}_{h}$. We recall from [4, Form. (4.18)] (see also [18, Lemma 3.8] for similar arguments) the formula, for $i=1$ and 2 ,

$$
\mu_{i} \sum_{K \in \mathcal{T}_{\boldsymbol{a}}} \int_{K}\left(\operatorname{grad} u_{i h}\right)(\boldsymbol{x}) \cdot\left(\operatorname{grad} \varphi_{\boldsymbol{a}}\right)(\boldsymbol{x}) \mathrm{d} \boldsymbol{x}=\int_{D_{\boldsymbol{a}}}\left(\operatorname{div} \boldsymbol{t}_{i h}\right)(\boldsymbol{x}) \mathrm{d} \boldsymbol{x}
$$


We also use the expansion: $\lambda_{h}=\sum_{\boldsymbol{a} \in \mathcal{V}_{h}} \lambda_{\boldsymbol{a}} \chi_{\boldsymbol{a}}$. Next, for each $\boldsymbol{a}$ in $\mathcal{V}_{h}$, we derive by combining (2.15) with (3.24) the equivalent formulation of equations (2.16) (this makes use of the fact that the integral of $\varphi_{\boldsymbol{a}}$ on $\omega$ is equal to meas $\left.\left(D_{\boldsymbol{a}}\right)\right)$ :

$$
\begin{aligned}
& \lambda_{\boldsymbol{a}} \operatorname{meas}\left(D_{\boldsymbol{a}}\right)=\int_{D_{\boldsymbol{a}}}\left(\operatorname{div} \boldsymbol{t}_{1 h}\right)(\boldsymbol{x}) \mathrm{d} \boldsymbol{x}-\int_{D_{\boldsymbol{a}}} f_{1 h}(\boldsymbol{x}) \mathrm{d} \boldsymbol{x}-\int_{\Delta_{\boldsymbol{a}}}\left(f_{1}-f_{1 h}\right)(\boldsymbol{x}) \varphi_{\boldsymbol{a}}(\boldsymbol{x}) \mathrm{d} \boldsymbol{x}, \\
& \lambda_{\boldsymbol{a}} \operatorname{meas}\left(D_{\boldsymbol{a}}\right)=-\int_{D_{\boldsymbol{a}}}\left(\operatorname{div} \boldsymbol{t}_{2 h}\right)(\boldsymbol{x}) \mathrm{d} \boldsymbol{x}+\int_{D_{\boldsymbol{a}}} f_{2 h}(\boldsymbol{x}) \mathrm{d} \boldsymbol{x}+\int_{\Delta_{\boldsymbol{a}}}\left(f_{2}-f_{2 h}\right)(\boldsymbol{x}) \varphi_{\boldsymbol{a}}(\boldsymbol{x}) \mathrm{d} \boldsymbol{x} .
\end{aligned}
$$

Noting that $\lambda_{\boldsymbol{a}} \operatorname{meas}\left(D_{\boldsymbol{a}}\right)$ is equal to $\int_{D_{\boldsymbol{a}}} \lambda_{h}(\boldsymbol{x}) d \boldsymbol{x}$, multiplying these equations by the mean value $\bar{\zeta}_{1}^{a}$ or $\bar{\zeta}_{2}^{a}$ of $\zeta_{1}$ or $\zeta_{2}$ on $D_{a}$ and subtracting the first one from the second one, we obtain

$$
\sum_{i=1}^{2} \int_{D \boldsymbol{a}}\left(f_{i h}-\operatorname{div} \boldsymbol{t}_{i h}-(-1)^{i} \lambda_{h}\right)(\boldsymbol{x}) \bar{\zeta}_{i}^{\boldsymbol{a}} \mathrm{d} \boldsymbol{x}=-\sum_{i=1}^{2} \int_{\Delta_{\boldsymbol{a}}}\left(f_{i}-f_{i h}\right)(\boldsymbol{x}) \bar{\zeta}_{i}^{\boldsymbol{a}} \varphi_{\boldsymbol{a}}(\boldsymbol{x}) d \boldsymbol{x} .
$$

Thus, we derive

$$
\begin{aligned}
& \sum_{i=1}^{2} \int_{D \boldsymbol{a}}\left(f_{i h}-\operatorname{div} \boldsymbol{t}_{i h}-(-1)^{i} \lambda_{h}\right)(\boldsymbol{x}) \zeta_{i}(\boldsymbol{x}) \mathrm{d} \boldsymbol{x} \\
&=\sum_{i=1}^{2}\left(\int_{D_{\boldsymbol{a}}}\left(f_{i h}-\operatorname{div} \boldsymbol{t}_{i h}-(-1)^{i} \lambda_{h}\right)(\boldsymbol{x})\left(\zeta_{i}(\boldsymbol{x})-\bar{\zeta}_{i}^{\boldsymbol{a}}\right) \mathrm{d} \boldsymbol{x}\right. \\
&\left.-\int_{\Delta_{\boldsymbol{a}}}\left(f_{i}-f_{i h}\right)(\boldsymbol{x})\left(\bar{\zeta}_{i}^{\boldsymbol{a}} \varphi_{\boldsymbol{a}}-\zeta_{i h}\right)(\boldsymbol{x}) d \boldsymbol{x}\right) .
\end{aligned}
$$

To bound the first term in the right-hand side, we use the Poincaré-Wirtinger inequality (3.3). To bound the second one, we sum on the $\boldsymbol{a}$, add and subtract $\zeta_{i}$, and use the approximation properties of its Clément-type interpolate $\sum_{\boldsymbol{a} \in \mathcal{V}_{h}} \bar{\zeta}_{i}^{a} \varphi_{\boldsymbol{a}}$ (note that $\zeta_{i}$ vanishes on $\partial \omega$ ) and also of $\zeta_{i h}$ (see once more [7, Thm 3.1.6]). When combined with (3.23), this gives

$$
\begin{aligned}
& \sum_{i=1}^{2} \int_{\omega}\left(f_{i h}-\operatorname{div}\right.\left.\boldsymbol{t}_{i h}-(-1)^{i} \lambda_{h}\right)(\boldsymbol{x}) \zeta_{i}(\boldsymbol{x}) \mathrm{d} \boldsymbol{x} \\
& \leq \sum_{i=1}^{2} \sum_{D \in \overline{\mathcal{D}}_{h}}\left(c_{D} h_{D} \mu_{i}^{-\frac{1}{2}}\left\|f_{i h}-\operatorname{div} \boldsymbol{t}_{i h}-(-1)^{i} \lambda_{h}\right\|_{L^{2}(D)}\right. \\
&\left.\quad+c \mu_{i}^{-\frac{1}{2}} h_{D}\left\|f_{i}-f_{i h}\right\|_{L^{2}(D)}\right)\left\|\mu_{i}^{\frac{1}{2}} \operatorname{grad} \zeta_{i}\right\|_{L^{2}(D)^{2}} .
\end{aligned}
$$

To conclude, we observe that

$$
-b\left(\boldsymbol{u}_{h}, \lambda_{h}\right)=\frac{1}{2} \sum_{D \in \overline{\mathcal{D}}_{h}} \eta_{D}^{(\mathrm{c})}
$$

By inserting this last equation, (3.21), (3.22), and (3.26) into (3.20) and using appropriate Cauchy-Schwarz inequalities, we obtain

$\left\|\tilde{\boldsymbol{u}}-\boldsymbol{u}_{h}\right\|^{2} \leq\left(\sum_{D \in \overline{\mathcal{D}}_{h}} \sum_{i=1}^{2}\left(\eta_{i D}^{(\mathrm{df})}+\eta_{i D}^{(\mathrm{r})}+c \mu_{i}^{-\frac{1}{2}} h_{D}\left\|f_{i}-f_{i h}\right\|_{L^{2}(D)}\right)^{2}\right)^{\frac{1}{2}}\left\|\tilde{\boldsymbol{u}}-\boldsymbol{u}_{h}\right\|+\frac{1}{2} \sum_{D \in \overline{\mathcal{D}}_{h}} \eta_{D}^{(\mathrm{c})}$. 
Using the inequality $a b \leq \frac{1}{2}\left(a^{2}+b^{2}\right)$ yields the desired estimate.

Theorem 3.4. Assume that the data $\left(f_{1}, f_{2}\right)$ belong to $L^{2}(\omega) \times L^{2}(\omega)$ and that the domain $\omega$ is convex. Then, the following a posteriori error estimate holds between the solutions $\boldsymbol{u}=\left(u_{1}, u_{2}\right)$ of problem $(2.7)$ and $\boldsymbol{u}_{h}=\left(u_{1 h}, u_{2 h}\right)$ of problem (2.11)

$$
\begin{array}{r}
\left\|\boldsymbol{u}-\boldsymbol{u}_{h}\right\| \leq\left(\sum_{D \in \overline{\mathcal{D}}_{h}}\left(\sum_{i=1}^{2}\left(\eta_{i D}^{(\mathrm{df})}+\eta_{i D}^{(\mathrm{r})}+c \mu_{i}^{-\frac{1}{2}} h_{D}\left\|f_{i}-f_{i h}\right\|_{L^{2}(D)}\right)^{2}+\eta_{D}^{(\mathrm{c})}\right)\right)^{\frac{1}{2}} \\
+c^{\prime}\left(\left\|g-g_{h}\right\|_{H^{\frac{1}{2}(\partial \omega)}}+\sqrt{\rho\left(f_{1}, f_{2}\right)}\left\|g-g_{h}\right\|_{H_{*}^{\frac{1}{2}}}^{-\frac{1}{2}(\partial \omega)}\right) .
\end{array}
$$

We are also in a position to prove an upper bound for the error $\left\|\lambda-\lambda_{h}\right\|_{H^{-1}(\Omega)}$.

Corollary 3.5. If the assumptions of Theorem 3.4 are satisfied, the following a posteriori error estimate holds between the solutions $\left(u_{1}, u_{2}, \lambda\right)$ of problem $(2.4)$ and $\left(u_{1 h}, u_{2 h}, \lambda_{h}\right)$ of problem (2.17)

$$
\begin{aligned}
& \left\|\lambda-\lambda_{h}\right\|_{H^{-1}(\omega)} \\
& \leq 2 \max \left\{\mu_{1}^{\frac{1}{2}}, \mu_{2}^{\frac{1}{2}}\right\}\left(\sum_{D \in \overline{\mathcal{D}}_{h}}\left(\sum_{i=1}^{2}\left(\eta_{i D}^{(\mathrm{df})}+\eta_{i D}^{(\mathrm{r})}+c \mu_{i}^{-\frac{1}{2}} h_{D}\left\|f_{i}-f_{i h}\right\|_{L^{2}(D)}\right)^{2}+\eta_{D}^{(\mathrm{c})}\right)\right)^{\frac{1}{2}} \\
& +c^{\prime}\left(\left\|g-g_{h}\right\|_{H^{\frac{1}{2}}(\partial \omega)}+\sqrt{\rho\left(f_{1}, f_{2}\right)}\left\|g-g_{h}\right\|_{H_{*}^{-\frac{1}{2}}(\partial \omega)}^{\frac{1}{2}}\right) .
\end{aligned}
$$

Proof: It follows from the definition of the norm of $H^{-1}(\omega)$ that

$$
\left\|\lambda-\lambda_{h}\right\|_{H^{-1}(\omega)}=\sup _{\boldsymbol{v} \in H_{0}^{1}(\omega)^{2}} \frac{b\left(\boldsymbol{v}, \lambda-\lambda_{h}\right)}{|\boldsymbol{v}|_{H^{1}(\omega)^{2}}} .
$$

Setting $\boldsymbol{v}=\left(v_{1}, v_{2}\right)$, we have

$$
b\left(\boldsymbol{v}, \lambda-\lambda_{h}\right)=\sum_{i=1}^{2}\left\langle f_{i}, v_{i}\right\rangle-a(\boldsymbol{u}, \boldsymbol{v})-b\left(\boldsymbol{v}, \lambda_{h}\right)
$$

whence

$$
b\left(\boldsymbol{v}, \lambda-\lambda_{h}\right)=\sum_{i=1}^{2}\left\langle f_{i}, v_{i}\right\rangle-a\left(\boldsymbol{u}_{h}, \boldsymbol{v}\right)-b\left(\boldsymbol{v}, \lambda_{h}\right)-a\left(\boldsymbol{u}-\boldsymbol{u}_{h}, v\right) .
$$

Evaluating the first three terms in the right hand-side of this equation follows exactly the same lines as in the proof of Lemma 3.3, with $\boldsymbol{\zeta}$ replaced by $\boldsymbol{v}$ (which also vanishes on $\partial \omega$ ), while the last term obviously satisfies

$$
a\left(\boldsymbol{u}-\boldsymbol{u}_{h}, \boldsymbol{v}\right) \leq\left\|\boldsymbol{u}-\boldsymbol{u}_{h}\right\| \max \left\{\mu_{1}^{\frac{1}{2}}, \mu_{2}^{\frac{1}{2}}\right\}|\boldsymbol{v}|_{H^{1}(\omega)^{2}}
$$

Combining all this with Theorem 3.4 gives the desired estimate. 


\subsection{Upper bounds for the indicators.}

We now intend to establish an upper bound of all indicators as a function of the local error. We first make complete the definition of the $\boldsymbol{t}_{i h}$, which is needed for computing the indicators $\eta_{i D}^{(\mathrm{df})}$ and $\eta_{i D}^{(\mathrm{r})}$ but also for proving the next estimates. From now, we work with the functions $\boldsymbol{t}_{i h}$ satisfying (3.2) and also, on each $e$ of $\mathcal{E}_{h} \backslash \mathcal{E}_{h}^{*}$ which is an edge of the elements $K$ and $K^{\prime}$ of $\mathcal{T}_{h}$,

$$
\left.\left(\boldsymbol{t}_{i h} \cdot \boldsymbol{n}\right)\right|_{e}=-\frac{\mu_{i}}{2}\left(\partial_{n}\left(\left.u_{i h}\right|_{K}\right)+\left(\partial_{n}\left(\left.u_{i h}\right|_{K^{\prime}}\right)\right)\right. \text {. }
$$

It follows from [15] that these equations define the $\boldsymbol{t}_{i h}$ in a unique way.

Remark 3.6. In the next proofs, we use several times the following property that we prefer to recall once: If two closed domains $o_{1}$ and $o_{2}$ have disjoint interiors and are such that the intersection $\partial o_{1} \cap \partial o_{2}$ has a positive measure, every distribution $\varphi$ in $H^{-1}\left(o_{1} \cup o_{2}\right)$ satisfies

$$
\|\varphi\|_{H^{-1}\left(o_{1}\right)}+\|\varphi\|_{H^{-1}\left(o_{2}\right)} \leq \sqrt{2}\|\varphi\|_{H^{-1}\left(o_{1} \cup o_{2}\right)} .
$$

Proposition 3.7. For $i=1$ and 2, the following bound holds for any indicator $\eta_{i D_{\boldsymbol{a}}}^{(\mathrm{df})}$ defined in (3.6), $\boldsymbol{a} \in \overline{\mathcal{V}}_{h}$ :

$$
\begin{aligned}
\eta_{i D \boldsymbol{a}}^{(\mathrm{df})} \leq c\left(\left\|\mu_{i}^{\frac{1}{2}} \operatorname{grad}\left(u_{i}-u_{i h}\right)\right\|_{L^{2}\left(\Delta_{\boldsymbol{a}}\right)^{2}}+\| \lambda\right. & -\lambda_{h} \|_{H^{-1}\left(\Delta_{\boldsymbol{a}}\right)} \\
& \left.+\sum_{K \in \mathcal{T}_{\boldsymbol{a}}} h_{K}\left\|f_{i}-f_{i h}\right\|_{L^{2}(K)}\right) .
\end{aligned}
$$

Proof: We only prove this bound for $i=1$ since its analogue for $i=2$ relies on exactly the same arguments. By switching to the reference triangle and using the Piola transform [9, Chap. III, Form. (4.63)], we easily derive that

$$
\forall \boldsymbol{s}_{h} \in \mathcal{R} \mathcal{T}(\kappa), \quad\left\|\boldsymbol{s}_{h}\right\|_{L^{2}(\kappa)^{2}} \leq c h_{\kappa}^{\frac{1}{2}}\left\|\boldsymbol{s}_{h} \cdot \boldsymbol{n}\right\|_{L^{2}(\partial \kappa)}
$$

Applying this formula to $\boldsymbol{s}_{h}=\mu_{1}^{\frac{1}{2}} \operatorname{grad} u_{1 h}+\mu_{1}^{-\frac{1}{2}} \boldsymbol{t}_{1 h}$ (since grad $u_{1 h}$ is constant on $\kappa$, it belongs to $\mathcal{R} \mathcal{T}(\kappa)$ ), we obtain

$$
\eta_{1 D \boldsymbol{a}}^{(\mathrm{df})} \leq c \sum_{\kappa \in \mathcal{S}_{h}, \kappa \subset D \boldsymbol{a}} h_{\kappa}^{\frac{1}{2}}\left\|\left(\mu_{1}^{\frac{1}{2}} \operatorname{grad} u_{1 h}+\mu_{1}^{-\frac{1}{2}} \boldsymbol{t}_{1 h}\right) \cdot \boldsymbol{n}\right\|_{L^{2}(\partial \kappa)}
$$

Note from (3.2) and (3.29) that $\left(\mu_{1}^{\frac{1}{2}} \operatorname{grad} u_{1 h}+\mu_{1}^{-\frac{1}{2}} \boldsymbol{t}_{1 h}\right) \cdot \boldsymbol{n}$ vanishes on all edges $e$ in $\mathcal{E}_{h}^{*}$ and is equal to $\frac{\mu_{1}}{2}$ times the jump of $\partial_{n} u_{1 h}$ on edges of $\mathcal{E}_{h} \backslash \mathcal{E}_{h}^{*}$. Denoting by $\mathcal{L}_{\boldsymbol{a}}$ the set of edges of elements of $\mathcal{T}_{h}$ that contain $\boldsymbol{a}$, this yields

$$
\eta_{1 D \boldsymbol{a}}^{(\mathrm{df})} \leq c \sum_{\ell \in \mathcal{L}_{\boldsymbol{a}}} h_{\ell}^{\frac{1}{2}}\left\|\mu_{1}^{\frac{1}{2}}\left[\partial_{n} u_{1 h}\right]_{\ell}\right\|_{L^{2}(\ell)}
$$

where $h_{\ell}$ denotes the length of $\ell$ and $[\cdot]_{\ell}$ the jump through $\ell$. To bound these last terms, we write the residual of the Laplace equation associated with $u_{1}-u_{1 h}$. It reads, for any 
$v$ in $H_{0}^{1}(\Omega)$ :

$$
\begin{aligned}
& \mu_{1} \int_{\omega} \operatorname{grad}\left(u_{1}-u_{1 h}\right)(\boldsymbol{x}) \cdot(\operatorname{grad} v)(\boldsymbol{x}) \mathrm{d} \boldsymbol{x} \\
& =\sum_{K \in \mathcal{T}_{h}}\left(\int_{K}\left(f_{1 h}+\lambda_{h}\right)(\boldsymbol{x}) v(\boldsymbol{x}) \mathrm{d} \boldsymbol{x}+\int_{K}\left(f_{1}-f_{1 h}\right)(\boldsymbol{x}) v(\boldsymbol{x}) \mathrm{d} \boldsymbol{x}+\int_{K}\left(\lambda-\lambda_{h}\right)(\boldsymbol{x}) v(\boldsymbol{x}) \mathrm{d} \boldsymbol{x}\right. \\
& \left.+\frac{1}{2} \sum_{\ell \in \mathcal{L}_{K}} \int_{\ell} \mu_{1}\left[\partial_{n} u_{1}\right]_{\ell}(\tau) v(\tau) d \tau\right)
\end{aligned}
$$

where $\mathcal{L}_{K}$ denotes the set of edges of $K$ which are not contained in $\partial \omega$. Thus, fully standard arguments (see $[17, \S 1.2]$ ) lead first to the estimate (note that $\Delta u_{1 h}$ is zero on each $K$ )

$$
\begin{aligned}
h_{K}\left\|f_{1 h}+\mu_{1} \Delta u_{1 h}+\lambda_{h}\right\|_{L^{2}(K)} \leq c(\| & \mu_{1} \operatorname{grad}\left(u_{1}-u_{1 h}\right) \|_{L^{2}(K)^{2}} \\
& \left.+\left\|\lambda-\lambda_{h}\right\|_{H^{-1}(K)}+h_{K}\left\|f_{1}-f_{1 h}\right\|_{L^{2}(K)}\right),
\end{aligned}
$$

and second, if $\ell$ is shared by two elements $K$ and $K^{\prime}$,

$$
\begin{array}{r}
h_{\ell}^{\frac{1}{2}}\left\|\mu_{1}^{\frac{1}{2}}\left[\partial_{n} u_{1 h}\right]_{\ell}\right\|_{L^{2}(\ell)} \leq c\left(\left\|\mu_{1}^{\frac{1}{2}} \operatorname{grad}\left(u_{1}-u_{1 h}\right)\right\|_{L^{2}\left(K \cup K^{\prime}\right)^{2}}+\left\|\lambda-\lambda_{h}\right\|_{H^{-1}\left(K \cup K^{\prime}\right)}\right. \\
\left.+h_{K}\left\|f_{1}-f_{1 h}\right\|_{L^{2}(K)}+h_{K^{\prime}}\left\|f_{1}-f_{1 h}\right\|_{L^{2}\left(K^{\prime}\right)}\right) .
\end{array}
$$

By inserting this last estimate into (3.32), we obtain the desired bound.

Proposition 3.8. For $i=1$ and 2 , the following bound holds for any indicator $\eta_{i D a}^{(\mathrm{r})}$ defined in (3.7), $\boldsymbol{a} \in \overline{\mathcal{V}}_{h}$ :

$$
\begin{aligned}
\eta_{i D \boldsymbol{a}}^{(\mathrm{r})} \leq c\left(\left\|\mu_{i}^{\frac{1}{2}} \operatorname{grad}\left(u_{i}-u_{i h}\right)\right\|_{L^{2}\left(\Delta_{\boldsymbol{a}}\right)^{2}}+\|\right. & -\lambda_{h} \|_{H^{-1}\left(\Delta_{\boldsymbol{a}}\right)} \\
& \left.+\sum_{K \in \mathcal{T}_{\boldsymbol{a}}} h_{K}\left\|f_{i}-f_{i h}\right\|_{L^{2}(K)}\right) .
\end{aligned}
$$

Proof: There also, we only prove this bound for $i=1$. We first observe that

$$
\eta_{1 D \boldsymbol{a}}^{(\mathrm{r})} \leq c\left(\sum_{K \subset \mathcal{T}_{\boldsymbol{a}}} h_{K} \mu_{1}^{-1}\left\|f_{1 h}-\operatorname{div} \boldsymbol{t}_{1 h}+\lambda_{h}\right\|_{L^{2}(K)}^{2}\right)^{\frac{1}{2}}
$$

Next we use the triangle inequality

$\left\|f_{1 h}-\operatorname{div} \boldsymbol{t}_{1 h}+\lambda_{h}\right\|_{L^{2}(K)} \leq\left\|f_{1 h}+\mu_{1} \Delta u_{1 h}+\lambda_{h}\right\|_{L^{2}(K)}+\mu_{1}^{\frac{1}{2}}\left\|\mu_{1}^{\frac{1}{2}} \Delta u_{1 h}+\mu_{i}^{-\frac{1}{2}} \operatorname{div} \boldsymbol{t}_{i h}\right\|_{L^{2}(K)}$.

The first term is bounded in (3.33), while evaluating the second one relies on a standard inverse inequality and (3.31).

Proposition 3.9. The following bound holds for any non-zero indicator $\eta_{D}^{(\mathrm{c})}$ defined in (3.8), $D \in \overline{\mathcal{D}}_{h}$ :

$$
\eta_{D}^{(\mathrm{c})} \leq c \sigma\left(f_{1}, f_{2}, g\right)\left(\left\|u_{1}-u_{1 h}\right\|_{L^{2}(D)}+\left\|u_{2}-u_{2 h}\right\|_{L^{2}(D)}+\left\|\lambda-\lambda_{h}\right\|_{H^{-1}(D)}\right) .
$$


where the constant $\sigma\left(f_{1}, g_{2}, g\right)$ is given for $s>0$ by

$$
\sigma\left(f_{1}, f_{2}, g\right)=\left\|f_{1}\right\|_{H^{-1}(\omega)}+\left\|f_{2}\right\|_{H^{-1}(\omega)}+\|g\|_{H^{\frac{1}{2}+s}(\partial \omega)}
$$

Proof: Assume that $\eta_{D}^{(\mathrm{c})}$ is not zero. Thus $D$ belongs to $\mathcal{D}_{h}$. Since both $\lambda_{h}$ and $u_{1 h}-u_{2 h}$ are nonnegative on $D$, it follows from [17, Lemma 3.3] that, if $\psi_{D}$ is the "bubble" function which is affine on each element $\kappa$ of $\mathcal{S}_{h}$ contained in $D$, is equal to 1 in the internal vertex $\boldsymbol{a}$ of $D$ and vanishes on $\partial D$,

$$
\eta_{D}^{(\mathrm{c})} \leq c \int_{D}\left(u_{1 h}-u_{2 h}\right)(\boldsymbol{x}) \lambda_{h}(\boldsymbol{x}) \psi_{D}(\boldsymbol{x}) d \boldsymbol{x} .
$$

Thus, using the third line of problem (1.1), we derive

$$
\begin{aligned}
\eta_{D}^{(\mathrm{c})} \leq c\left(\left|\int_{D}\left(u_{1 h}-u_{2 h}\right)(\boldsymbol{x})\left(\lambda-\lambda_{h}\right)(\boldsymbol{x}) \psi_{D}(\boldsymbol{x}) d \boldsymbol{x}\right|\right. \\
\left.+\left|\int_{D}\left(\left(u_{1}-u_{2}\right)-\left(u_{1 h}-u_{2 h}\right)\right)(\boldsymbol{x}) \lambda(\boldsymbol{x}) \psi_{D} d \boldsymbol{x}\right|\right) .
\end{aligned}
$$

This yields

$$
\begin{aligned}
& \eta_{D}^{(\mathrm{c})} \leq c\left(\left\|\lambda-\lambda_{h}\right\|_{H^{-1}(D)}\left|\left(u_{1 h}-u_{2 h}\right) \psi_{D}\right|_{H^{1}(D)}\right. \\
& \left.+\left(\left\|u_{1}-u_{1 h}\right\|_{L^{2}(D)}+\left\|u_{2}-u_{2 h}\right\|_{L^{2}(D)}\right)\left\|\lambda \psi_{D}\right\|_{L^{2}(D)}\right) .
\end{aligned}
$$

By switching to the reference element and noting from (3.9) that, if $D$ coincides with $D_{\boldsymbol{a}}$, $\left(u_{1 h}-u_{2 h}\right)(\boldsymbol{a})$ is zero, we obtain

$$
\left|\left(u_{1 h}-u_{2 h}\right) \psi_{D}\right|_{H^{1}(D)} \leq c\left|u_{1 h}-u_{2 h}\right|_{H^{1}(D)} .
$$

It follows from standard arguments that, for any $s>0$,

$$
\left|u_{1 h}\right|_{H^{1}(\omega)}+\left|u_{2 h}\right|_{H^{1}(\omega)} \leq c\left(\left\|f_{1}\right\|_{H^{-1}(\omega)}+\left\|f_{2}\right\|_{H^{-1}(\omega)}+\|g\|_{H^{\frac{1}{2}+s}(\partial \omega)}\right) .
$$

Inserting this and the bound for $\|\lambda\|_{L^{2}(\omega)}$ stated in (2.5) into (3.37) leads to the desired estimate.

Remark 3.10. The same arguments as previously yield that, in estimate (3.35), each quantity $\left\|u_{i}-u_{i h}\right\|_{L^{2}(D)}, i=1$ and 2 , can be replaced by $h_{D}\left|u_{i}-u_{i h}\right|_{H^{1}(D)}$, whence the modified estimate

$$
\eta_{D}^{(\mathrm{c})} \leq c \sigma\left(f_{1}, f_{2}, g\right)\left(h_{D}\left|u_{1}-u_{1 h}\right|_{H^{1}(D)}+h_{D}\left|u_{2}-u_{2 h}\right|_{H^{1}(D)}+\left\|\lambda-\lambda_{h}\right\|_{H^{-1}(D)}\right) .
$$

Remark 3.11. It must be noted that the constants $c$ which appear in (3.31), (3.34) and (3.35) only depend on the constants $c_{D}^{(P W)}$ and $c_{D}^{(P F)}$ which are introduced in (3.3) and (3.4), respectively, on the coefficents $\mu_{1}$ and $\mu_{2}$, and also on the regularity parameter $\sigma$ of the family of triangulations $\left(\mathcal{T}_{h}\right)_{h}$. We have not made this dependence explicit for simplicity.

\subsection{Conclusions.}


Let us assume for a while that the $f_{i h}$ coincide with the $f_{i}$ and that $g_{h}$ coincides with $g$. In this case, estimates (3.27) and (3.28) read

$$
\begin{aligned}
\left\|\boldsymbol{u}-\boldsymbol{u}_{h}\right\|+\frac{1}{2} \min \left\{\mu_{1}^{-\frac{1}{2}}, \mu_{2}^{-\frac{1}{2}}\right\}\left\|\lambda-\lambda_{h}\right\|_{H^{-1}(\omega)} & \\
& \leq 2\left(\sum_{D \in \overline{\mathcal{D}}_{h}}\left(\sum_{i=1}^{2}\left(\eta_{i D}^{(\mathrm{df})}+\eta_{i D}^{(\mathrm{r})}\right)^{2}+\eta_{D}^{(\mathrm{c})}\right)\right)^{\frac{1}{2}} .
\end{aligned}
$$

It must be noted that no unknown constant appears in this estimate. Moreover, when compared with the local estimates stated in Proposition 3.7 and 3.8

$$
\max \left\{\eta_{i D \boldsymbol{a}}^{(\mathrm{df})}, \eta_{i D_{\boldsymbol{a}}}^{(\mathrm{r})}\right\} \leq c\left(\| \mu_{i}^{\frac{1}{2}} \operatorname{grad}\left(u_{i}-u_{i h}\left\|_{L^{2}\left(\Delta_{\boldsymbol{a}}\right)^{2}}+\right\| \lambda-\lambda_{h} \|_{H^{-1}\left(\Delta_{\boldsymbol{a}}\right)}\right)\right.
$$

it appears that error indicators $\eta_{i D \boldsymbol{a}}^{(\mathrm{df})}$ and $\eta_{i \boldsymbol{a}_{\boldsymbol{a}}}^{(\mathrm{r})}$ are optimal quantities for evaluating the error in a sharp way. On the other hand, when comparing (3.35) or (3.38) with (3.40), the indicators $\eta_{D}^{(\mathrm{c})}$ are not fully optimal according to the criteria for variational equations, but our results seem the best possible ones for variational inequalities (see [3, Thms 7.2 \& $7.5]$ and $[11, \S 4]$ for very similar results in different frameworks). In any case, numerical experiments below confirm the observation of Remark 3.1 that these indicators are negligible. Moreover estimates (3.31), (3.34) and (3.35) are local, in the sense that each indicator associated with a domain $D$ is bounded by the error in a small neighbourhood of $D$. So, these indicators should provide an efficient tool for mesh adaptivity.

Remark 3.12. It can be seen from the proof of Lemma 3.3 that the a posteriori error estimates (3.27) and (3.28) hold for any $\boldsymbol{t}_{i h}$ satisfying (3.2) only on the edges $e$ of $\mathcal{E}_{h}^{*}$ which lie on the boundary of some $D$ in $\mathcal{D}_{h}$. On the other hand, the following choice of $\boldsymbol{t}_{i h}$ is proposed in [18]:

1) $\boldsymbol{t}_{i h} \cdot \boldsymbol{n}$ is given by (3.2) on such edges $e$ of $\mathcal{E}_{h}^{*}$ which lie on the boundary of some $D$ in $\mathcal{D}_{h}$

2) $\boldsymbol{t}_{i h} \cdot \boldsymbol{n}$ on the edges which lie in the interior of some $D$ in $\overline{\mathcal{D}}_{h}$ or on the boundary $\partial \omega$ is given so that

$$
\begin{aligned}
& \lambda_{\boldsymbol{a}} \operatorname{meas}(\kappa)=\int_{\kappa}\left(\operatorname{div} \boldsymbol{t}_{1 h}\right)(\boldsymbol{x}) \mathrm{d} \boldsymbol{x}-\int_{\kappa} f_{1 h}(\boldsymbol{x}) \mathrm{d} \boldsymbol{x}-\int_{K^{\kappa}}\left(f_{1}-f_{1 h}\right)(\boldsymbol{x}) \varphi_{\boldsymbol{a}}(\boldsymbol{x}) \mathrm{d} \boldsymbol{x}, \\
& \lambda_{\boldsymbol{a}} \operatorname{meas}(\kappa)=-\int_{\kappa}\left(\operatorname{div} \boldsymbol{t}_{2 h}\right)(\boldsymbol{x}) \mathrm{d} \boldsymbol{x}+\int_{\kappa} f_{2 h}(\boldsymbol{x}) \mathrm{d} \boldsymbol{x}+\int_{K^{\kappa}}\left(f_{2}-f_{2 h}\right)(\boldsymbol{x}) \varphi_{\boldsymbol{a}}(\boldsymbol{x}) \mathrm{d} \boldsymbol{x}
\end{aligned}
$$

for all $\kappa$ in $\mathcal{S}_{h}$ contained in $D_{\boldsymbol{a}}$. Here the elements $K^{\kappa}$ denote triangles forming a partition of $\Delta_{\boldsymbol{a}}$ obtained by dividing each $K$ in $\mathcal{T}_{\boldsymbol{a}}$ into two triangles by joining $\boldsymbol{a}$ and the opposite edge midpoint and each $K^{\kappa}$ contains $\kappa$. It follows from $[18, \S 7]$ that such $\boldsymbol{t}_{i h}$ exist and can be constructed locally in each $\overline{\mathcal{D}}_{h}$ by only prescribing the given degrees of freedom (fluxes through the edges). In particular, no (local) linear system solution is necessary. Even if we do not prove the analogues of estimates (3.31) and (3.34) for the indicators associated with these modified $\boldsymbol{t}_{i h}$, numerical experiments [18] indicate that they are sharper than the previous ones (i.e., the constant $c$ in (3.31) and (3.34) should be smaller). For this reason, we make this new choice of the $\boldsymbol{t}_{i h}$ in our numerical calculations. 


\section{Numerical experiments.}

The numerical simulations that we now present have been performed using the code FreeFem ++ due to Hecht and Pironneau, see [10]. In all cases, the implementation is made as follows:

- We first solve the reduced discrete problem (2.11) via the primal-dual active set strategy. Details on this algorithm can be found in [12].

- Next, we compute the action $\lambda_{h}$ as a solution of a linear system with diagonal matrix. More precisely, it follows from (2.16) that the coefficients $\lambda_{\boldsymbol{a}}, \boldsymbol{a} \in \mathcal{V}_{h}$, are given by

$$
\lambda_{\boldsymbol{a}} \operatorname{meas}\left(D_{\boldsymbol{a}}\right)=\mu_{1} \int_{\omega}\left(\operatorname{grad} u_{1 h}\right)(\boldsymbol{x}) \cdot\left(\operatorname{grad} \varphi_{\boldsymbol{a}}\right)(\boldsymbol{x}) \mathrm{d} \boldsymbol{x}-\int_{\omega} f_{1}(\boldsymbol{x}) \varphi_{\boldsymbol{a}}(\boldsymbol{x}) \mathrm{d} \boldsymbol{x} .
$$

Note that only the mesh $\mathcal{T}_{h}$ is involved in this problem, so that mesh adaptivity can be realized via standard methods.

Remark 4.1. A still less expensive way of computing $u_{1 h}$ and $u_{2 h}$ relies on the following observation: When setting $u_{+}=\mu_{1} u_{1}+\mu_{2} u_{2}$ and $u_{-}=u_{1}-u_{2}$, problem (1.1) is equivalent to the system

$$
\begin{cases}-\Delta u_{+}=f_{1}+f_{2} & \text { in } \omega, \\ u_{+}=\mu_{1} g & \text { on } \partial \omega,\end{cases}
$$

and

$$
\begin{cases}-\Delta u_{-}-\left(\frac{1}{\mu_{1}}+\frac{1}{\mu_{2}}\right) \lambda=\frac{1}{\mu_{1}} f_{1}-\frac{1}{\mu_{2}} f_{2} & \text { in } \omega \\ u_{-} \geq 0, \quad \lambda \geq 0, \quad \lambda u_{-}=0 & \text { in } \omega, \\ u_{-}=g & \text { on } \partial \omega\end{cases}
$$

where the two unknowns $u_{+}$and $u_{-}$are completely uncoupled. Of course, we use these new unknowns for the computation. But we have preferred to present the analysis of the discretization in the initial formulation, in view of the extensions to more than two membranes and also to the contact between shells.

Remark 4.2. In the implementation of the discretization, each term $\left\langle f_{i}, v_{i h}-u_{i h}\right\rangle$ in problem (2.11) is as usual replaced by $\left\langle f_{i h}, v_{i h}-u_{i h}\right\rangle$. In this case, the constant $c$ which appears in (3.27) is simply the Poincaré-Wirtinger constant $c_{D}^{(P W)}$.

Mesh adaptivity is performed according to the following simple strategy:

- Initialization step: We first choose the initial mesh $\mathcal{T}_{h}^{0}$ such that the errors on the data $\left\|f_{i}-f_{i h}\right\|_{L^{2}(\omega)}$ and $\left\|g-g_{h}\right\|_{H^{\frac{1}{2}(\partial \omega)}}$ are smaller than a given tolerance $\eta^{*}$.

- Adaptation step: Given the triangulation $\mathcal{T}_{h}^{n}$, we compute the solution $\left(u_{1 h}^{n}, u_{2 h}^{n}, \lambda_{h}^{n}\right)$, next the corresponding indicators $\eta_{i D}^{(\mathrm{df})}, \eta_{i D}^{(\mathrm{r})}$, and $\eta_{D}^{(\mathrm{c})}$ together with their sum $\eta_{D}$. Then we construct a new triangulation $\mathcal{T}_{h}^{n+1}$ such that the diameter of any element in $\mathcal{D}_{h}^{n+1}$ (with obvious notation) which contains or is contained in an element $D$ of $\mathcal{D}_{h}^{n}$ is proportional to the diameter of $D$ times the ratio $\bar{\eta} / \eta_{D}$, where $\bar{\eta}$ is the mean value of the $\eta_{D}$. We refer to [8, Chap. 21] for the way of constructing such a mesh.

Of course, the adaptation step is iterated either a fixed number of times or until the total estimate becomes smaller than the tolerance $\eta^{*}$. 


\subsection{Case of a given solution.}

In a first step, we work with a given solution in a disk in order to check the good convergence properties of the discretization and the efficiency of the error indicators. Let $\omega$ denote the disk with centre $(0,0)$ and radius 1 . We use the polar coordinates $(r, \theta)$ on $\omega$.

We take $\mu_{1}$ and $\mu_{2}$ equal to 1 for simplicity. The datum $g$ on the boundary is now a positive constant. We consider the triple $\left(u_{1}, u_{2}, \lambda\right)$ given by, for $0 \leq \theta \leq 2 \pi$,

$$
\begin{gathered}
u_{1}(r, \theta)=g\left(2 r^{2}-1\right), \quad 0 \leq r \leq 1, \\
u_{2}(r, \theta)= \begin{cases}g\left(2 r^{2}-1\right), & 0 \leq r \leq \frac{1}{\sqrt{2}}, \\
g(1-r)\left(2 r^{2}-1\right) \frac{\sqrt{2}}{\sqrt{2}-1}, & \frac{1}{\sqrt{2}} \leq r \leq 1,\end{cases} \\
\lambda(r, \theta)= \begin{cases}2 g, & 0 \leq r \leq \frac{1}{\sqrt{2}}, \\
0, & \frac{1}{\sqrt{2}} \leq r \leq 1 .\end{cases}
\end{gathered}
$$

Equivalently, this triple coincides with the solution of system (1.1) for the data $f_{1}$ and $f_{2}$ given by

$$
\begin{gathered}
f_{1}(r, \theta)= \begin{cases}-10 g, & 0 \leq r \leq \frac{1}{\sqrt{2}}, \\
-8 g, & \frac{1}{\sqrt{2}} \leq r \leq 1,\end{cases} \\
f_{2}(r, \theta)= \begin{cases}-6 g, & 0 \leq r \leq \frac{1}{\sqrt{2}}, \\
-g \frac{1+8 r-18 r^{2}}{r} \frac{\sqrt{2}}{\sqrt{2}-1}, & \frac{1}{\sqrt{2}} \leq r \leq 1 .\end{cases}
\end{gathered}
$$

The displacements $u_{1}$ and $u_{2}$ of the membranes in the plane $y=0$, with $g$ equal to 0.05 , are presented in Figure 1.

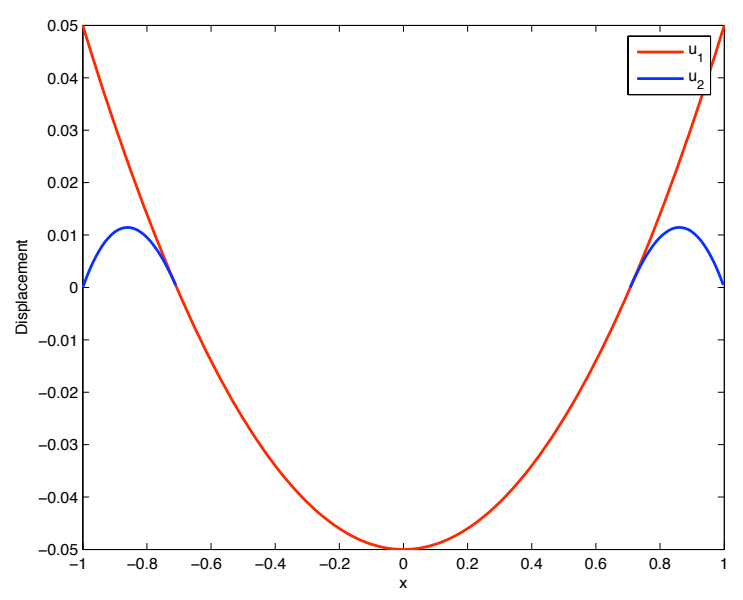

Figure 1. Displacements of the two membranes

Only for this experiment, we do not perform the initialization step in the adaptivity process, in order to bring to light the influence of the discontinuities of the data $f_{1}$ and $f_{2}$ on the computation. Note that a particular attention had to be paid so that the meshes approximate correctly, with increasing level of refinement, the two circles $r=1$ and $r=\frac{1}{\sqrt{2}}$. Thus, since the action $\lambda$ is only non-zero on the disk $r \leq \frac{1}{\sqrt{2}}$ and constant on this disk, 
the error $\left\|\lambda-\lambda_{h}\right\|_{H^{-1}(\omega)}$ is negligible in comparison of $\left\|\boldsymbol{u}-\boldsymbol{u}_{h}\right\|$, so that we omit it on the next figures.

Denoting by $\beta_{h}$ the cardinality of $\overline{\mathcal{V}}_{h}$, i.e., the number of vertices of all $K$ in $\mathcal{T}_{h}$, we first present in the left part of Figure 2 the curves of the error $\left\|\boldsymbol{u}-\boldsymbol{u}_{h}\right\|$ and different estimators as a function of $\beta_{h}$ in a sequence of uniformly refined meshes. In particular, the $\mathcal{O}(h)$ a priori error estimate of Section 2 is confirmed: The experimental order of convergence, defined as

$$
\frac{\log e_{h}^{n}-\log e_{h}^{h-1}}{\frac{1}{2}\left(\log \beta_{h}^{n-1}-\log \beta_{h}^{n}\right)},
$$

where $e_{h}^{n}$ is the error $\left\|\boldsymbol{u}-\boldsymbol{u}_{h}\right\|$ on the mesh $\mathcal{T}_{h}^{n}$ and with obvious notation for the $\beta_{h}^{n}$, is here close to 1 .
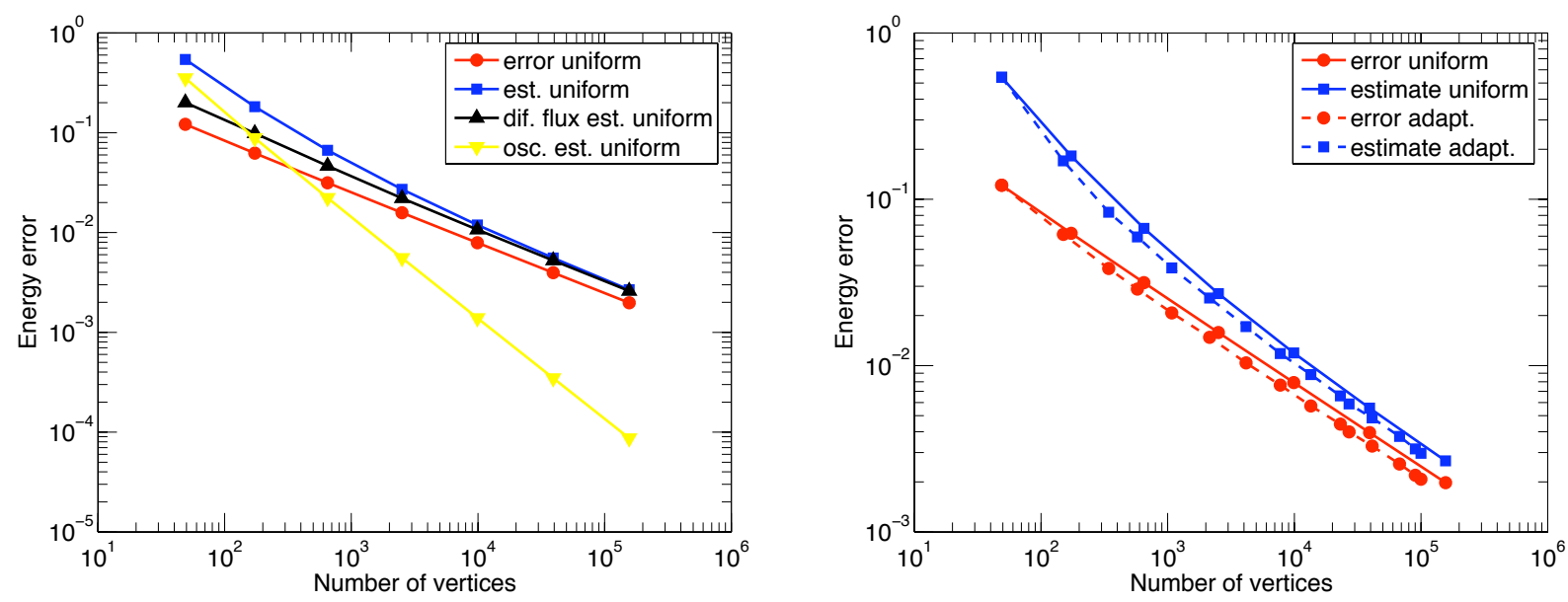

Figure 2. Actual and estimated errors

The right part of Figure 2 presents the same curves but now both for uniform and adapted meshes. It can be observed that, since the solution is regular, the gain due to mesh adaptivity is very weak. We give the precise values of the errors and the convergence order for different values of $\beta_{h}$ in Table 1 , for uniformly refined meshes.

\begin{tabular}{|c|c|c|}
\hline Number of vertices & $\left\|\boldsymbol{u}-\boldsymbol{u}_{h}\right\|$ & Convergence order \\
\hline & & \\
2549 & 0.0315 & 1.0341 \\
9889 & 0.0158 & 1.0198 \\
39233 & 0.0079 & 1.0108 \\
156289 & 0.0040 & 1.0056 \\
& 0.0020 & 1.0029 \\
\hline
\end{tabular}

Table 1. Error and convergence order as a function of $\beta_{h}$

We also clearly see that the a posteriori error estimator $\eta_{h}$ in Theorem 3.4, namely 
the quantity

$$
\eta_{h}=\left(\sum_{D \in \overline{\mathcal{D}}_{h}}\left(\sum_{i=1}^{2}\left(\eta_{i D}^{(\mathrm{df})}+\eta_{i D}^{(\mathrm{r})}+c_{D}^{(P W)} h_{D}\left\|f_{i}-f_{i h}\right\|_{L^{2}(D)}\right)^{2}+\eta_{D}^{(\mathrm{c})}\right)\right)^{\frac{1}{2}},
$$

provides an upper bound for the error $\left\|\boldsymbol{u}-\boldsymbol{u}_{h}\right\|$. This is illustrated in Figure 3, where the corresponding effectivity index, i.e., the ratio $\eta_{h} /\left\|\boldsymbol{u}-\boldsymbol{u}_{h}\right\|$, is given both for uniformly refined and adapted meshes.

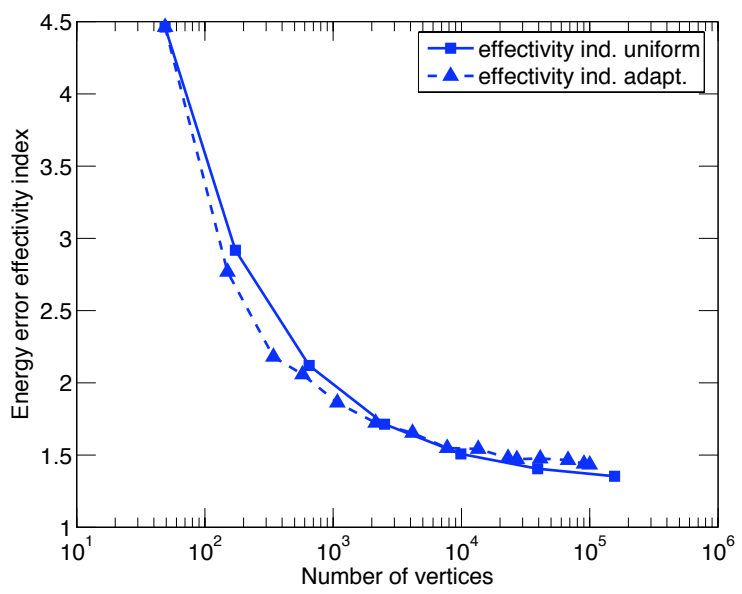

Figure 3. Effectivity index as a function of $\beta_{h}$

Moreover, it can be observed that, even on a coarse mesh (nearly uniform mesh with $\beta_{h}=173$ ), the local error indicators provide a good evaluation of the error distribution, see Figure 4 where the distribution of the error is presented in the left part and the error indicators are presented in the right part.
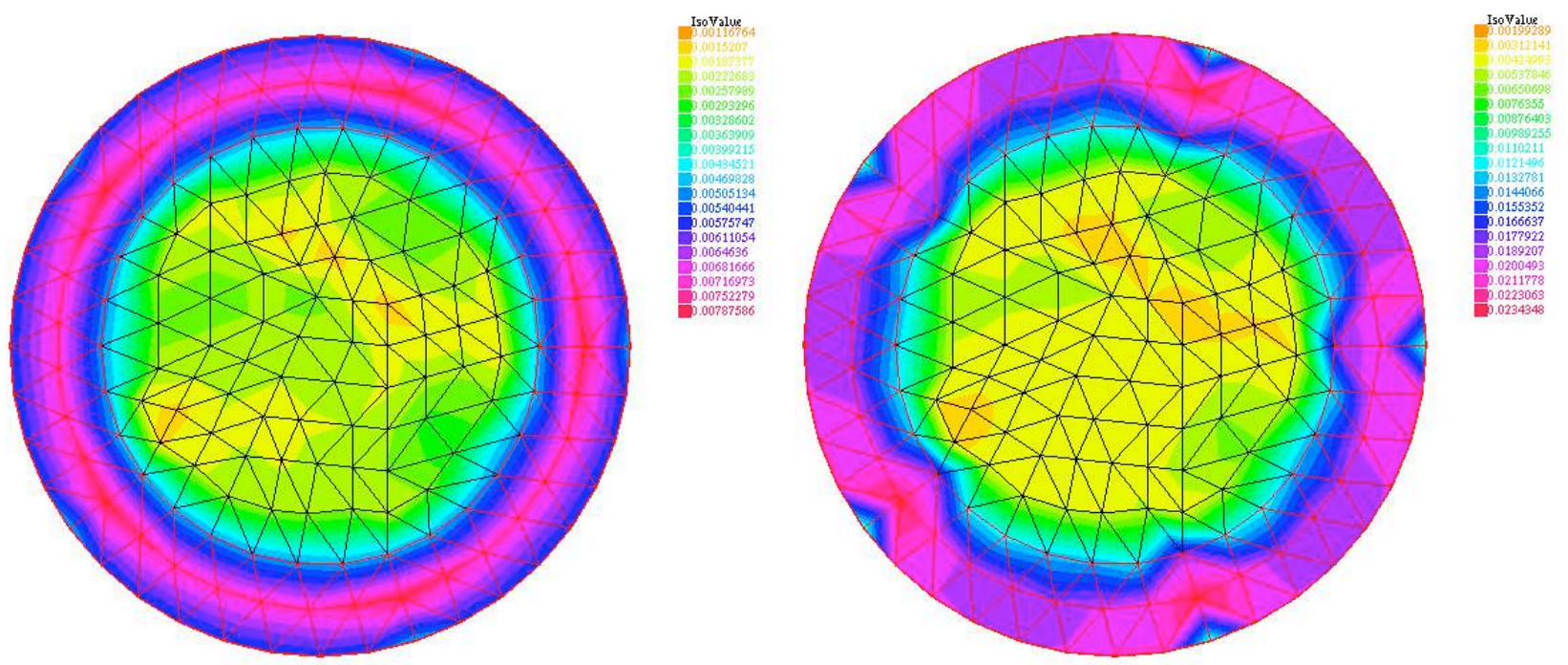

Figure 4. Actual and estimated error distributions

To conclude with this experiment, we briefly describe the comparative behaviours of the four parts of $\eta_{h}$, obviously denoted by $\eta_{h}^{(\mathrm{df})}, \eta_{h}^{(\mathrm{r})}, \varepsilon_{h}^{(\mathrm{d})}$ for the term involving the data $f_{1}$ and $f_{2}$, and $\eta_{h}^{(\mathrm{c})}$.

(i) For coarse meshes, the data oscillation estimator $\varepsilon_{h}^{(\mathrm{d})}$ dominates the other ones but, 
since the $f_{i}$ are pieewise regular, $\varepsilon_{h}^{(\mathrm{d})}$ converges by one order faster and gets negligible for fine meshes. We note that the local quantities $c_{D}^{(P W)} h_{D}\left\|f_{i}-f_{i h}\right\|_{L^{2}(D)}$ are only nonzero in the second membrane and for $r>1 / \sqrt{2}$. This is also the reason for the increased value of the effectivity index on coarse meshes and its rapid decrease towards the optimal value of one.

(ii) The crucial contribution of $\eta_{h}$ turns out to be given by $\eta_{h}^{(\mathrm{df})}$.

(iii) The contact error estimator $\eta_{h}^{(\mathrm{c})}$ is four orders of magnitude smaller than $\eta_{h}$ on coarse meshes and it decays very rapidly.

(iv) The residual error estimators $\eta_{h}^{(\mathrm{r})}$ are zero thanks to the chosen construction of the equilibrated fluxes proposed in Remark 3.12.

Finally, it can be checked that the lack of optimality of the estimator $\eta_{h}^{(\mathrm{c})}$ (see Section 3.4) is negligible: Indeed, all indicators $\eta_{D}^{(\mathrm{c})}$ are smaller than $10^{-21}$.

\subsection{Case of unknown solutions.}

We now make use of the adaptivity strategy described at the beginning of this section to compute the adapted mesh and the corresponding solution in the two following cases.

(i) The domain $\omega$ is the rectangle

$$
\omega=] 0,2[\times] 0,1[.
$$
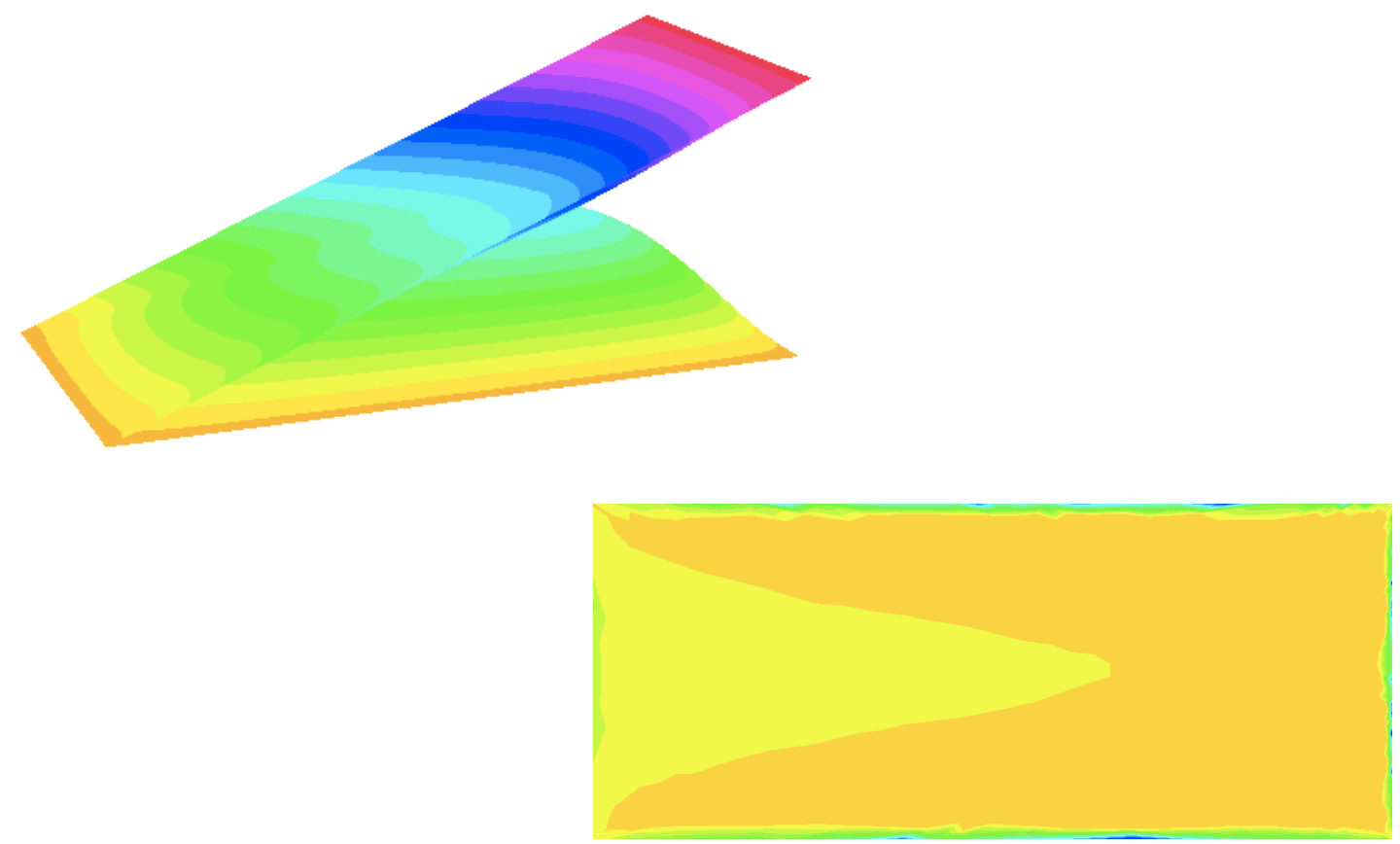

Figure 5. The displacements and action in the case (4.10) - (4.11)

We now take $\mu_{1}$ equal to 1 and $\mu_{2}$ equal to 5 . The data $f_{1}, f_{2}$ are constant, but the boundary condition $g$ is not. More precisely, we take

$$
f_{1}(x, y)=-1 \quad \text { and } \quad f_{2}(x, y)=0 \quad \text { on } \omega, \quad g(x, y)=0.1 \times x \quad \text { on } \partial \omega .
$$

It can be noted that, before displacements, the membranes are in contact on the edge $x=0$ but not elsewhere. Even these geometry and data are very simple, they correspond to several realistic situations. 
Figure 5 presents the displacements $u_{1 h}$ and $u_{2 h}$ (top part) and also the isovalues of the action $\lambda_{h}$ (bottom part) for the geometry in (4.10) and the data in (4.11). Note that the contact zone is easy to identify from these drawings.

(ii) The domain $\omega$ is now not convex:

$$
\Omega=] 0,3[\times] 0,1[\bigcup] 1,2[\times[1,2[.
$$

The coefficients $\mu_{1}$ and $\mu_{2}$ are both equal to 1 . The three data $f_{1}, f_{2}$, and $g$ are now given by

$$
\begin{gathered}
f_{1}(x, y)=0 \quad \text { on } \omega \quad \text { and } \quad f_{2}(x, y)= \begin{cases}5 & \text { if }(x, y) \in] \frac{5}{4}, \frac{7}{4}[\times] \frac{1}{4}, \frac{3}{4}[, \\
0 & \text { elsewhere }\end{cases} \\
g(x, y)=0.05 \quad \text { on } \partial \omega .
\end{gathered}
$$

Figure 6 presents the same surfaces and curves as Figure 5, but now for the geometry in (4.12) and the data in (4.13). Figure 7 presents the adapted mesh (after 10 iterations of the adaptation step), also for these new data.
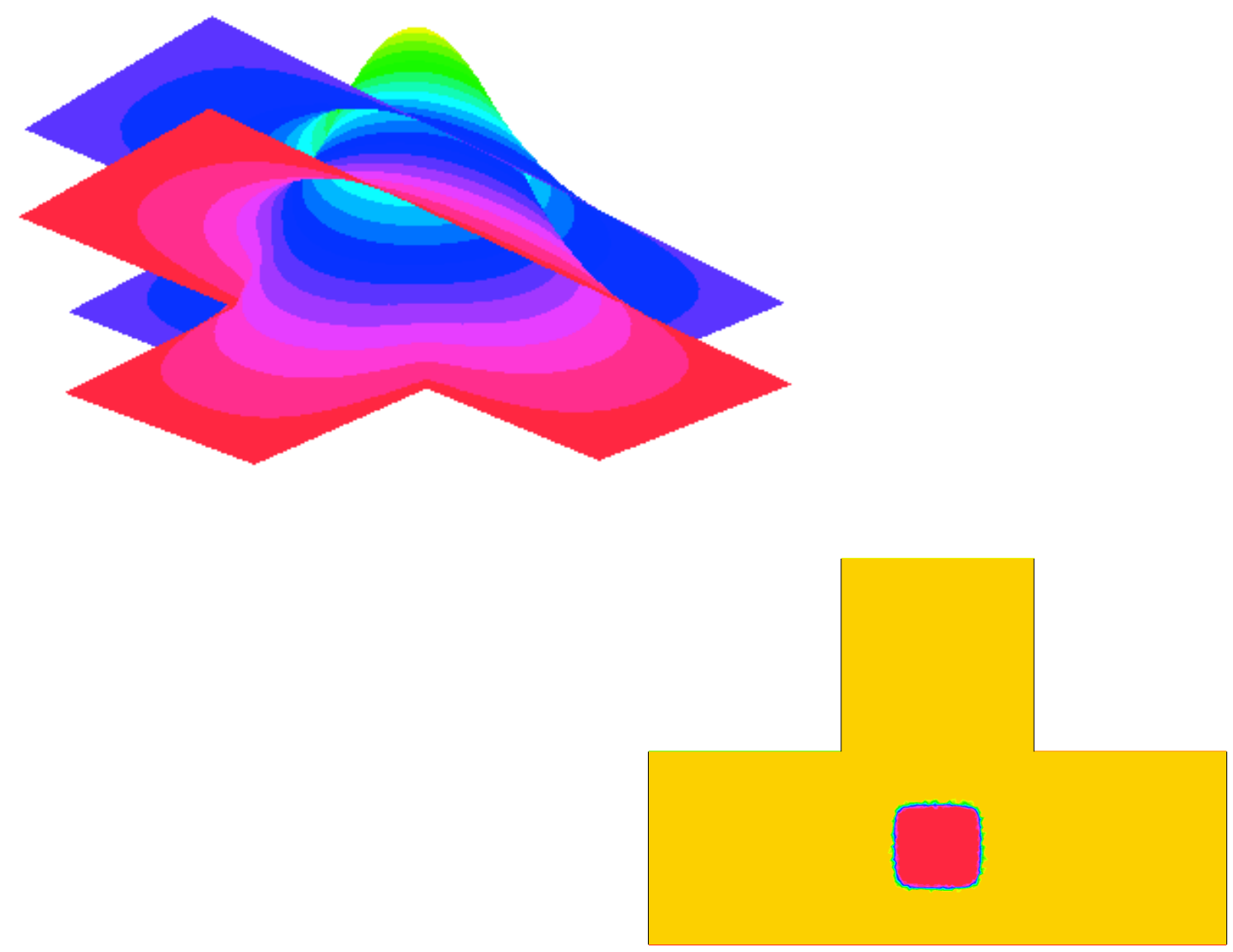

Figure 6. The displacements and action in the case $(4.12)-(4.13)$ 


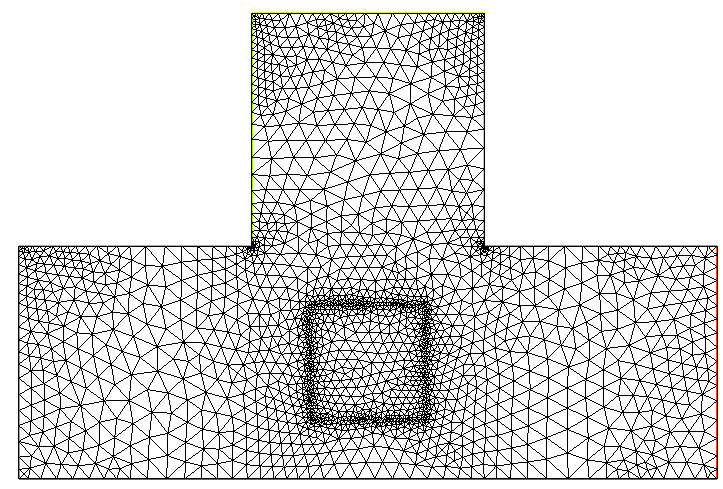

Figure 7. The adapted mesh in the case (4.12) - (4.13) with $\mu_{1}=\mu_{2}=1$

To conclude, we present the adapted mesh, for the same data in (4.13) and again after 10 iterations of the adaptation step, but now with $\mu_{1}$ equal to 1 and $\mu_{2}$ equal to 5 . Indeed, it appears from several computations that identifying the contact zone is more difficult with different tension coefficients, see problem (4.3).

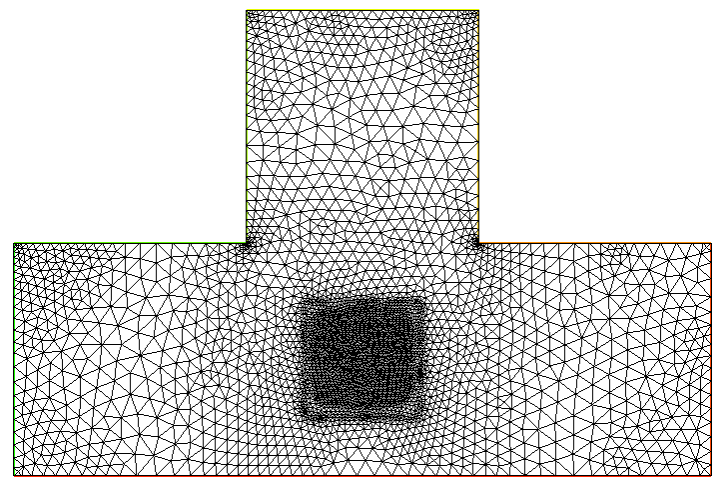

Figure 8. The adapted mesh in the case (4.12) - (4.13) with $\mu_{1}=1$ and $\mu_{2}=5$ 


\section{References}

[1] M. Ainsworth, J.T. Oden, C.Y. Lee - Local a posteriori error estimators for variational inequalities, Numer. Methods for Partial Differential Equations 9 (1993), 23-33.

[2] Z. Belhachmi, F. Hecht, S. Tahir - Adaptive finite elements for variational inequalities with nonsmooth coefficients. The case of bounded contrast in the coefficients, submitted for publication.

[3] F. Ben Belgacem, C. Bernardi, A. Blouza, M. Vohralík - A finite element discretization of the contact between two membranes, Math. Model. Numer. Anal. 43 (2009), 33-52.

[4] F. Ben Belgacem, C. Bernardi, A. Blouza, M. Vohralík - On the unilateral contact between membranes, Part 1: Finite element discretization and mixed reformulation, Math. Model. Nat. Phenom. 4 (2009), 21-43.

[5] D. Braess, R.H.W. Hoppe, J. Schöberl - A posteriori estimators for obstacle problems by the hypercircle method, Comput. Vis. Sci. 11 (2008), 351-362.

[6] Z. Chen, R.H. Nochetto - Residual type a posteriori error estimates for elliptic obstacle problems, Numer. Math. 84 (2000), 527-548.

[7] P.G. Ciarlet - The Finite Element Method for Elliptic Problems, North-Holland, Amsterdam, New-York, Oxford (1978).

[8] P.J. Frey, P.-L. George - Maillages, applications aux éléments finis, Hermès (1999).

[9] V. Girault, P.-A. Raviart — Finite Element Methods for Navier-Stokes Equations, Theory and Algorithms, Springer-Verlag (1986).

[10] F. Hecht, O. Pironneau - FreeFem++, see www.freefem.org.

[11] P. Hild, S. Nicaise - Residual a posteriori error estimators for contact problems in elasticity, Math. Model. Numer. Anal. 41 (2007), 897-923.

[12] M. Hintermüller, K. Ito, K. Kunisch - The primal-dual active set strategy as a semismooth Newton method, SIAM J. Optim. 13 (2002), 865-888.

[13] I. Hlaváček, J. Haslinger, J. Nečas, J. Lovíšek — Solution of Variational Inequalities in Mechanics, Applied Mathematical Sciences 66, Translated from the Slovak by J. Jarník, Springer-Verlag (1988).

[14] R.H. Nochetto, K.G. Siebert, A. Veeser - Pointwise a posteriori error control for elliptic obstacle problems, Numer. Math. 95 (2003), 163-195.

[15] P.-A. Raviart, J.-M. Thomas - A mixed finite element method for second order elliptic problems, in Mathematical Aspects of Finite Element Methods, Lecture Notes in Mathematics 606, Springer (1977), 292-315.

[16] S.I. Repin — Functional a posteriori estimates for elliptic variational inequalities, J. Math. Sciences 152 (2008), 702-712.

[17] R. Verfürth - A Review of A Posteriori Error Estimation and Adaptive Mesh-Refinement Techniques, Wiley \& Teubner (1996).

[18] M. Vohralík - Guaranteed and fully robust a posteriori error estimates for conforming discretizations of diffusion problems with discontinuous coefficients, submitted for publication (2008).

[19] A. Weiss, B.I. Wohlmuth - A posteriori error estimator and error control for contact problems, Math. Comp. 78 (2009), 1237-1267.

[20] B.I. Wohlmuth - An a posteriori error estimator for two body contact problems on nonmatching meshes, J. Sci. Comput 33 (2007), 25-45. 